\title{
Role of primary excitation statistics in the generation of antibunched and sub-Poisson light
}

\author{
M. C. Teich, B. E. A. Saleh, and J. Peřina \\ Columbia Radiation Laboratory, Columbia University, New York, New York 10027.
}

Received December 5, 1983; accepted January 20, 1984

\begin{abstract}
We examine the coherence properties of stationary light obtained by the superposition of nonstationary independent emissions occurring at random space-time points. The positions of the points are independent and uniformly distributed over the volume of the source. The emission times fluctuate in accordance with a stationary renewal point process. This process admits of super-Poisson, sub-Poisson, or Poisson behavior. The individual emissions are assumed to be in a coherent, chaotic, or $n$ state. The statistical nature of the random-emission space-time points plays an important role in determining the coherence properties and photon statistics of the total field. This is manifested in the normalized second-order correlation function, which turns out to have the usual form for chaotic light with two additional terms. The first of these is determined by the statistical nature of the individual emissions (it is positive for coherent or chaotic, but zero for single-photon, emissions). The second term is governed by the statistics of the primary excitations (it is positive for super-Poisson, zero for Poisson, and negative for sub-Poisson excitations). Both additional terms become small for light with a high degeneracy parameter (many total photons per emission lifetime). The behavior of the light is then asymptotically chaotic. In the opposite limit, when the degeneracy parameter is small (or the emissions are instantaneous), the correlation properties of the primary excitations are directly transferred to the correlation properties of the photons. The first-order spatial-coherence properties of the field turn out to be identical with those of chaotic light (i.e., the van Cittert-Zernike theorem is obeyed), although the second-order properties differ. The photon-counting distribution reflects the character of the correlation function. Thus sub-Poisson primary excitations, together with $n$-state emissions, can lead to sub-Poisson photon counts under certain conditions. Such nonclassical light may be made arbitrarily intense if interference effects are eliminated by detecting many spatial modes. As an example of our theory, we cite a Franck-Hertz experiment excited by a space-charge-limited electron beam. If the electron excitations are represented as a sub-Poisson renewal point process, and the photon emissions as one-photon states, the light generated
should be antibunched and sub-Poisson.
\end{abstract}

\section{INTRODUCTION}

It is well known that under most circumstances, the statistical behavior of photon registrations can be described in terms of the doubly stochastic Poisson point process (DSPP). ${ }^{1}$ For such light, the ratio of photon-count variance $\operatorname{Var}(n)$ to photon-count mean $\langle n\rangle$, designated as the Fano factor $F_{n}(T)$, is $\geq 1$ for all choices of the counting-time interval $[0, T]$. For pure Poisson light, $F_{n}(T)=1$, independent of $T$. DSPP light is sometimes also called super-Poisson or bunched, since the photons tend to be emitted in bunches rather than strictly at random. ${ }^{2}$

It has long been recognized that under special circumstances, it is possible to generate so-called antibunched light, for which successive photon emissions are less likely than for Poisson light. ${ }^{3,4}$ When the condition $0 \leq F_{n}(T)<1$ is obeyed, the light is called sub-Poisson. We have recently elucidated the relationship between antibunched and sub-Poisson light, demonstrating that these two attributes of nonclassical light need not necessarily accompany each other. ${ }^{5}$ In addition, a source may or may not exhibit squeezing. 6 The measure $F_{n}(T)$ is useful because it succinctly describes the noisiness of a light source (in terms of noise-to-signal ratio) relative to that of an ideal amplitude-stabilized laser (coherent source), for which $F_{n}(T)=1$ for all $T$. Although ideal laser light has the useful property of exhibiting coherence to all orders, ${ }^{7}$ sub-Poisson light is less noisy in a photon-counting paradigm. Such quiet light may therefore find use in applications such as optical signal processing and optical communications. It could also serve as an improved source for investigating many physical and biological processes, such as the behavior of the human visual system at the threshold of seeing. ${ }^{8}$

In recent years, a number of optical processes have been proposed for generating antibunched light, including degenerate parametric amplification 4,9 and many other effects in which light interacts with a nonlinear medium. Mechanisms that have been considered include two-photon and multiphoton absorption, Raman and hyper-Raman scattering, the use of interference in parametric processes, and optical bistability and multistability. ${ }^{10}$ Viewed in an elementary way, these nonlinear optical processes operate by removing pairs (or clusters) of photons from the (Poisson) beam of exciting light, leaving behind an antibunched residue. The optical process of resonance fluorescence is, so far, the only phenomenon in which the antibunching effect has actually been observed. ${ }^{11}$ Kimble et al. ${ }^{11}$ argued that the observation of this process is a reflection of the fact that the atom makes a quantum jump to its ground state at the time a photon is emitted. The inability of the atom to radiate in the ground state may be viewed as an enforced dead time ${ }^{12}$ following photon emission, during which further emissions are prohibited. This regularizes the photon emissions from a single atom in resonance fluorescence, leading to antibunching and sub-Poisson behavior. ${ }^{13-15}$ For resonance fluorescence to operate in this way, however, there must be only a single atom in the field of view at any given time, because fluctuations in 


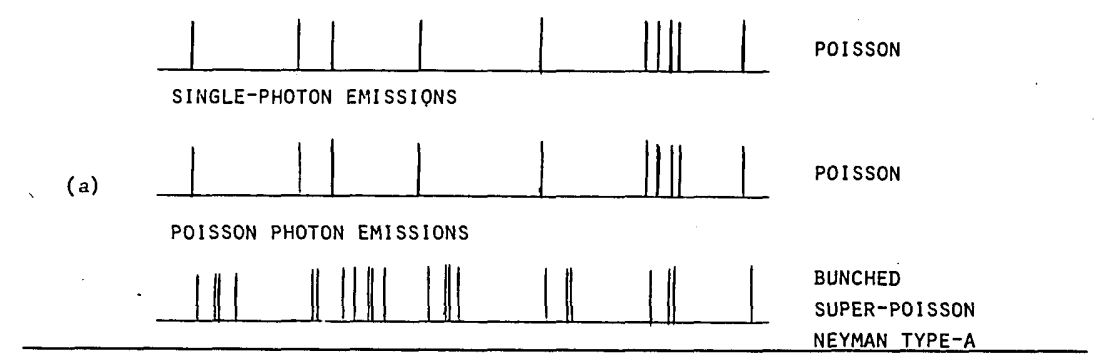

SUB-POISSON EXCITATIONS

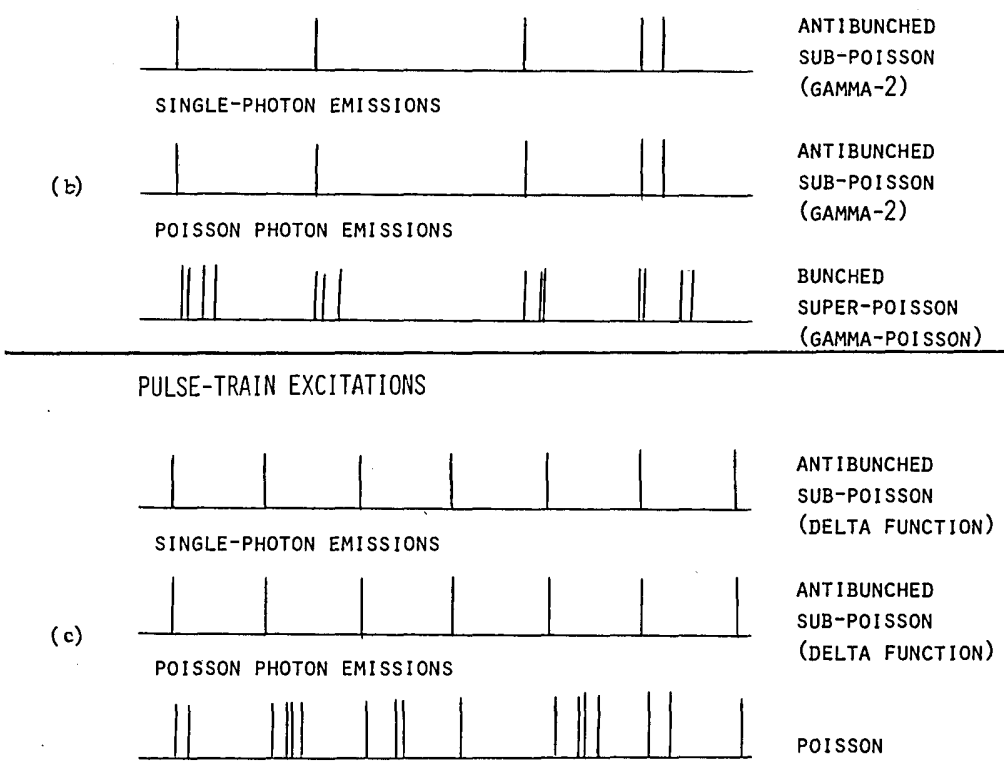

Fig. 1. Schematic representation of a two-step process for the generation of light, illustrating stochastic excitations (first line) with either instantaneous single-photon emission (second line) or Poisson multiple-photon emissions (third line). Interference effects are ignored in this simple representation. (a) Poisson excitations. (b) Antibunched, sub-Poisson excitations (gamma-2). (c) Pulse-train excitations (random phase).

the number of active radiators work against the desired effect. ${ }^{11,15-17}$

For light generated by a two-step process of excitation and emission, there are two key effects that regulate its antibunching and sub-Poisson possibilities: (1) the statistical properties of the excitations themselves and (2) the statistical properties of the individual emissions. This is most simply illustrated in terms of the schematic representation provided in Fig. 1. In Fig. 1(a), we show an excitation process that is Poisson. Consider each excitation as generating photons independently. Now if each excitation instantaneously produces a single photon, and if we ignore the effects of interference, the outcome is a Poisson stream of photons, which is neither antibunched nor, obviously, sub-Poisson. This is the least random situation that we could hope to produce, given the Poisson excitation statistics. Interference will redistribute the photon occurrences, leading us to the results for chaotic light (which is both bunched and super-Poisson). ${ }^{17}$ On the other hand, the individual nonstationary emissions may consist of multiple photons or random numbers of photons. In this case, we encounter two sources of randomness, one associated with the excitations and another associated with the emissions, so that the outcome will be both bunched and super-Poisson. In particular, if the emissions are also described by Poisson statistics, and the counting time is sufficiently long, we recover the Neyman Type-A counting distribution, as we have discussed in detail elsewhere. ${ }^{18}$ Even if the individual emissions are composed of deterministic numbers of photons, the end result is the fixed-multiplicative Poisson distribution, ${ }^{18}$ which is super-Poisson. Related results have been obtained when interference is permitted. ${ }^{17} \mathrm{It}$ is quite clear, therefore, that if the excitations themselves are Poisson (or super-Poisson), there is little hope of generating antibunched or sub-Poisson light by such a two-step process.

In Fig. 1(b) we consider a situation in which the excitations are more regular than those for the Poisson. For illustration and concreteness, we choose the excitation process to be produced by deleting every other event of a Poisson pulse train. The outcome is the gamma-2 (or Erlang-2) renewal process, whose analytical properties are well understood. ${ }^{19}$ Single-photon emissions, in the absence of interference, result 


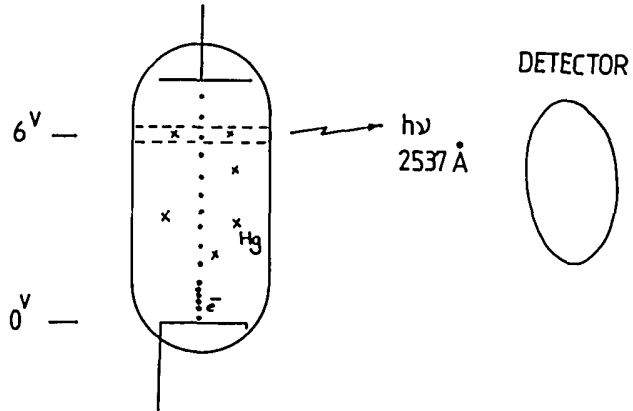

Fig. 2. Schematic representation of an experiment for generating antibunched and sub-Poisson Franck-Hertz light from the $6^{3} P_{1} \rightarrow$ $6^{1} S_{0}$ atomic transition in $\mathrm{Hg}$. The wavelength of the radiation is 2537 $\AA(h \nu=4.88 \mathrm{eV})$ in the near ultraviolet (see Ref. 5).

in antibunched, sub-Poisson photon statistics. Poisson emissions, on the other hand, result in super-Poisson light statistics. Of course, the presence of interference introduces additional bunching. Clearly, a broad variety of excitation processes can be invoked for generating many different kinds of light. A process that is similar to the gamma-2, and for which many analytical results are available, is the nonparalyzable dead-time-modified Poisson process. ${ }^{12,19}$ Resonance fluorescence radiation from a single atom will be described by a process of this type since, after emitting a single photon, the atom decays to the ground state where it cannot radiate. However, the superposition of light from a number of such atoms will wash out the sub-Poisson behavior.

Finally, in Fig. 1(c), we consider the case of pulse-train excitations (with random phase). This is the limiting result both for the gamma family of processes and for the deadtime-modified Poisson process. In the absence of interference, single-photon emissions in this case yield antibunched, ideally sub-Poisson photon statistics. Interference causes the antibunching to disappear, but the sub-Poisson nature remains in the long-counting-time limit. Poisson emissions give rise to Poisson photon statistics.

The illustration in Fig. 1 is intended to emphasize the importance of the excitation statistics as a determinant of the character of the generated light. To produce antibunched and/or sub-Poisson photons, both sub-Poisson excitations and sub-Poisson emissions are required. The production of sub-Poisson light by means of the nonlinear optical processes discussed above has been difficult because all these processes involve a struggle, in one way or another, to reduce the Poisson fluctuations of the exciting optical beam (which is usually derived from a laser).

This discussion provides the rationale underlying our recent suggestion ${ }^{5}$ for producing antibunched and sub-Poisson light by using the Franck-Hertz effect. Space charge is used to produce sub-Poisson electron excitations. Since the individual emissions are single photons, the generated light can be antibunched and sub-Poisson (see Fig. 2 for a schematic representation). This light can be strong if interference effects are minimized. ${ }^{20}$

Of course, once such light is produced by whatever means, there are obstacles to maintaining its character. These include optical absorption (random deletion) and the addition of spontaneous emission, both of which dilute (but fortunately do not destroy) the antibunching and sub-Poisson properties of the light. 21,22
In this paper, we study the effect of primary excitation statistics on the coherence and photon statistics of light. We account for the effects of temporal and spatial interference. Our results will have application in understanding the mechanisms associated with the generation of nonclassical light. Other areas in which our model may be useful are in the production of light from a random number of radiators 23,24 and in understanding non-Gaussian scattered light. ${ }^{17,25}$ In Section 2, we present the mathematical model. The coherence properties and photon statistics of the generated light are discussed in Sections 3 and 4, respectively. The conclusion is presented in Section 5 .

\section{MODEL}

Consider an optical field generated by a sequence of independent emissions at random times $\left\{t_{k}\right\}$ from radiators located at random positions $\left\{\mathbf{r}_{k}^{\prime}\right\}$ in a light source of volume $V$. This is illustrated schematically in Figs. 3 and 4.

The positive-frequency part of the field at position $\mathbf{r}$ and time $t$ may be written as a sum of independent components:

$$
\hat{E}^{+}(\mathbf{r}, t)=\sum_{k} v_{k}(\mathbf{r}, t) \hat{a}_{k}
$$

where $\hat{a}_{k}$ is the photon-annihilation operator for the $k$ th emission. Each emission is assumed to be describable by a single nonmonochromatic mode, ${ }^{7}$ whose space-time dependence is characterized by the function $v_{k}(\mathbf{r}, t)$. Furthermore, we assume that the functions $\left\{v_{k}(\mathbf{r}, t)\right\}$ are identical in shape but centered about different space-time points $\left\{\mathbf{r}_{k}^{\prime}, t_{k}\right\}$, i.e.,

$$
v_{k}(\mathbf{r}, t)=\epsilon\left(\mathbf{r}-\mathbf{r}_{k}^{\prime}, t-t_{k}\right) .
$$

The vector $\mathbf{r}_{k}^{\prime}$ represents the location at which the $k$ th emission is generated, and the time $t_{k}$ is the time at which it starts (see Figs. 3 and 4). These space-time points are assumed to be random points in a four-dimensional space. The times $\left\{t_{k}\right\}$ constitute a stationary random point process.

For simplicity we assume that $\epsilon(\mathbf{r}, t)$ is separable into a product of time-space functions:

$$
\epsilon(\mathbf{r}, t)=s(\mathbf{r}) \xi(t)
$$

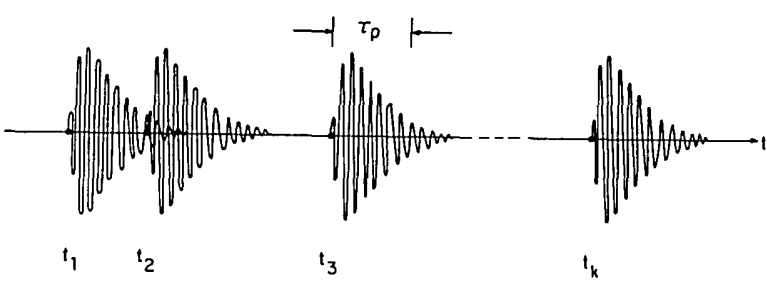

Fig. 3. Optical field emissions at the random times $\left\{t_{k}\right\}$.

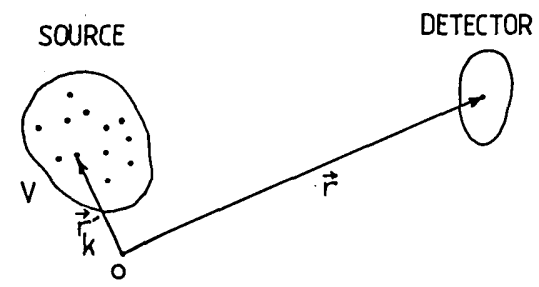

Fig. 4. Optical field emissions from radiators located at the random positions $\left\{\mathbf{r}_{k}^{\prime}\right\}$. 
The function $\xi(t)$ is taken to be a nonstationary function of time, but because the emission times $\left\{t_{k}\right\}$ are stationary, the total field is stationary.

We wish to determine the first- and second-order correlation functions of the field, ${ }^{3}$ denoted $G^{(1)}$ and $G^{(2)}$, respectively. We first calculate these functions conditioned on a realization of the emission times $\left\{t_{k}\right\}$, and the locations $\left\{\mathbf{r}_{k}^{\prime}\right\}$, of the radiators. To do this, we use the definitions

$$
\begin{aligned}
& G^{(1)}\left(\mathbf{r}_{1}, t ; \mathbf{r}_{2}, t+\tau \mid\left\{\mathbf{r}_{k}^{\prime}, t_{k}\right\}\right)=\operatorname{Tr}\left[\hat{\rho} \hat{E}^{-}\left(\mathbf{r}_{1}, t\right) \hat{E}^{+}\left(\mathbf{r}_{2}, t+\tau\right)\right], \\
& G^{(2)}\left(\mathbf{r}_{1}, t ; \mathbf{r}_{2}, t+\tau \mid\left\{\mathbf{r}_{k}^{\prime}, t_{k}\right\}\right)=\operatorname{Tr}\left[\hat{\rho} \hat{E}^{-}\left(\mathbf{r}_{1}, t\right)\right. \\
& \left.\quad \times \hat{E}^{-}\left(\mathbf{r}_{2}, t+\tau\right) \hat{E}^{+}\left(\mathbf{r}_{2}, t+\tau\right) \hat{E}^{+}\left(\mathbf{r}_{1}, t\right)\right],
\end{aligned}
$$

where $\hat{\rho}$ is the density operator of the field, $\hat{E}^{-}$is the negative-frequency part of the field, and $\tau$ represents the time difference. We shall subsequently average Eqs. (2.4) and (2.5) over the fluctuations of $\left\{t_{k}\right\}$ and $\left\{\mathbf{r}_{k}^{\prime}\right\}$, finally to obtain the correlation functions

$G^{(j)}\left(\mathbf{r}_{1}, \mathbf{r}_{2}, \tau\right)=\left\langle G^{(j)}\left(\mathbf{r}_{1}, t ; \mathbf{r}_{2}, t+\tau \mid\left\{\mathbf{r}_{k}^{\prime}, t_{k}\right\}\right)\right\rangle, \quad j=1,2$.

Here $\langle\cdot\rangle$ represents a classical ensemble average over the fluctuations of $\left\{\mathbf{r}_{k}^{\prime}, t_{k}\right\}$.

\section{A. Statistics of the Emission Times}

We assume that the emission times $\left\{t_{k}\right\}$ arise as a realization of a stationary renewal point process of rate $\mu$ (emissions per second). A renewal process is characterized by statistically independent, identically distributed interevent times. If $\tilde{\tau}$ is the interevent time (time between two successive emissions), then the probability density function $f(\tilde{\tau})$ completely defines a renewal process. In Appendix A, we review a number of important properties of renewal processes that we use in our development.

\section{B. Statistics of the Radiators' Positions}

We take the positions $\left\{\mathbf{r}_{k}^{\prime}\right\}$ of the radiators to be independent of the emission times $\left\{t_{k}\right\}$. The emissions are assumed to be statistically independent and uniformly distributed within the volume of the source $V$.

\section{States of the Individual Emissions}

The individual emissions are assumed to be statistically independent and statistically identical. Their state is defined by the density operator ${ }^{3}$

$$
\hat{\rho}=\prod_{k} \hat{\rho}_{k}
$$

We consider three important cases:

(i) Quasi-coherent (random phase) emissions for which

$$
\hat{\hat{\rho}}_{k}=\frac{1}{2 \pi} \int|\alpha\rangle\langle\alpha| \delta\left(|\alpha|-\left|\alpha_{0}\right|\right) \mathrm{d}^{2} \alpha,
$$

where $|\alpha\rangle$ is the coherent state and $\bar{n}_{0}=\left|\alpha_{0}\right|^{2}$ is the average number of photons per emission;

(ii) Thermal emissions for which

$$
\hat{\rho}_{k}=\int\left(1 / \pi \bar{n}_{0}\right) \exp \left(-|\alpha|^{2} / \bar{n}_{0}\right)|\alpha\rangle\langle\alpha| \mathrm{d}^{2} \alpha,
$$

with an average of $\bar{n}_{0}$ photons per emission; and (iii) n-state emissions for which

$$
\hat{\rho}_{k}=|n\rangle\langle n|,
$$

where $|n\rangle$ is the number state (Fock state) and the number of photons per emission is $\bar{n}_{0}=n$.

All the states considered above share the property that

$$
\left\langle\hat{a}_{k}^{\dagger} \hat{a}_{l}\right\rangle=\bar{n}_{0} \delta_{k, l}
$$

Furthermore, the expectation values of all odd-order combinations of $\hat{a}_{k}^{\dagger}$ and $\hat{a}_{k}$ vanish.

\section{CORRELATION FUNCTIONS}

\section{A. Conditional First- and Second-Order Correlation Functions}

The first- and second-order correlation functions defined in Eqs. (2.4) and (2.5) may be determined by substitution from Eq. (2.1), with the help of Eq. (2.11). This leads to

$$
G^{(1)}\left(\mathbf{r}_{1}, t ; \mathbf{r}_{2}, t+\tau \mid\left\{\mathbf{r}_{k}^{\prime}, \mathbf{t}_{k}\right\}\right)=\bar{n}_{0} \sum_{k} v_{k}^{*}\left(\mathbf{r}_{1}, t\right) v_{k}\left(\mathbf{r}_{2}, t+\tau\right),
$$

$$
\begin{aligned}
& G^{(2)}\left(\mathbf{r}_{1}, t ; \mathbf{r}_{2}, t+\tau \mid\left\{\mathbf{r}_{k}^{\prime}, t_{k}\right\}\right)=\bar{n}_{0}^{2} \\
& \quad \times\left\{\sum_{k}\left|v_{k}\left(\mathbf{r}_{1}, t\right)\right|^{2} \sum_{l}\left|v_{l}\left(\mathbf{r}_{2}, t+\tau\right)\right|^{2}\right. \\
& \quad+\left|\sum_{k} v_{k}^{*}\left(\mathbf{r}_{1}, t\right) v_{k}\left(\mathbf{r}_{2}, t+\tau\right)\right|^{2} \\
& \left.\quad-(2-\beta) \sum_{k}\left|v_{k} *\left(\mathbf{r}_{1}, t\right) v_{k}\left(\mathbf{r}_{2}, t+\tau\right)\right|^{2}\right\},
\end{aligned}
$$

where

$$
\beta=\left\langle\hat{a}_{k}^{\dagger} \hat{a}_{k}^{\dagger} \hat{a}_{k} \hat{a}_{k}\right\rangle /\left\langle\hat{a}_{k}^{\dagger} \hat{a}_{k}\right\rangle^{2}
$$

The value of $\beta$ is easily shown to depend on the state of the emissions as follows:

Quasi-coherent:

$$
\begin{aligned}
& \beta=1, \\
& \beta=2, \\
& \beta=1-\frac{1}{n_{0}},
\end{aligned}
$$$$
\text { (iii) } n \text {-state: }
$$

in particular, for the 1-state: $\quad \beta=0$.

We note that these values for $\beta$ are those usually given for the normalized second-order correlation function $g^{(2)}(0)$ for stationary light of each type. In our case, however, $\beta$ is associated with individual nonstationary emissions. These emis sions have yet to be averaged over the excitation statistics.

The parameters $\beta$ and $\bar{n}_{0}$ determine the variance of the number of photons per emission in accordance with the relation

$$
\operatorname{Var}\left(n_{0}\right)=\bar{n}_{0}+(\beta-1) \bar{n}_{0}^{2} .
$$

It is evident that, for quasi-coherent and thermal emissions, this variance turns out to be $\bar{n}_{0}$ and $\bar{n}_{0}+\bar{n}_{0}$, respectively, as expected. For $n$-state emissions, on the other hand, $\operatorname{Var}\left(n_{0}\right)$ $=0$. 
Teich et al.

\section{B. Averaging over Emission Times and Positions}

By using the properties of stationary filtered marked renewal processes presented in Appendix A, Eqs. (3.1) and (3.2) can be averaged over the fluctuations of $\left\{\mathbf{r}_{k}^{\prime}, t_{k}\right\}$. This gives rise to [see Eqs. (A16) and (A17)]

$$
\begin{aligned}
& G^{(1)}\left(\mathbf{r}_{1}, \mathbf{r}_{2}, \tau\right)=\bar{n}_{0} \mu \int_{-\infty}^{\infty} \Gamma_{1}\left(\mathbf{r}_{1}, t ; r_{2}, t+\tau\right) \mathrm{d} t \\
& G^{(2)}\left(\mathbf{r}_{1}, \mathbf{r}_{2}, \tau\right)=\bar{n}_{0}{ }^{2}\left\{\mu^{2} \int_{-\infty}^{\infty} \Gamma_{1}\left(\mathbf{r}_{1}, t ; \mathbf{r}_{1}, t\right) \mathrm{d} t\right. \\
& \quad \times \int_{-\infty}^{\infty} \Gamma_{1}\left(\mathbf{r}_{2}, t ; \mathbf{r}_{2}, t\right) \mathrm{d} t \\
& +\mu^{2}\left|\int_{-\infty}^{\infty} \Gamma_{1}\left(\mathbf{r}_{1}, t ; \mathbf{r}_{2}, t+\tau\right) \mathrm{d} t\right|^{2} \\
& \quad+\beta \mu \int_{-\infty}^{\infty} \Gamma_{2}\left(\mathbf{r}_{1}, t ; \mathbf{r}_{2}, t+\tau\right) \mathrm{d} t \\
& \quad+\mu^{2} \rho \gamma(\tau)
\end{aligned}
$$$$
\circledast \int_{-\infty}^{\infty} \Gamma_{1}\left(\mathbf{r}_{1}, t ; \mathbf{r}_{1}, t\right) \Gamma_{1}\left(\mathbf{r}_{2}, t+\tau ; \mathbf{r}_{2}, t+\tau\right) \mathrm{d} t
$$$$
+\mu^{2} \rho \int_{-\infty}^{\infty} \mathrm{d} t \gamma(t) \int_{-\infty}^{\infty} \Gamma_{1}\left(\mathbf{r}_{1}, t^{\prime} ; \mathbf{r}_{2}, t^{\prime}+\tau\right)
$$$$
\left.\times \Gamma_{1}\left(\mathbf{r}_{1}, t+t^{\prime} ; \mathbf{r}_{2}, t+t^{\prime}+\tau\right) \mathrm{d} t^{\prime}\right\}
$$

The spatial behavior of $G^{(1)}, g^{(1)}$, and $g^{(2)}$ is determined by the functions

$$
\begin{aligned}
& D^{(1)}\left(\mathbf{r}_{1}, \mathbf{r}_{2}\right)=\frac{1}{V} \int_{V} s^{*}\left(\mathbf{r}_{1}-\mathbf{r}^{\prime}\right) s\left(\mathbf{r}_{2}-\mathbf{r}^{\prime}\right) \mathrm{d} \mathbf{r}^{\prime} \\
& D^{(2)}\left(\mathbf{r}_{1}, \mathbf{r}_{2}\right)=\frac{1}{V} \int_{V}\left|s\left(\mathbf{r}_{1}-\mathbf{r}^{\prime}\right)\right|^{2}\left|s\left(\mathbf{r}_{2}-\mathbf{r}^{\prime}\right)\right|^{2} \mathrm{~d} \mathbf{r}^{\prime}
\end{aligned}
$$

with the normalizations

$$
\begin{aligned}
& d^{(1)}\left(\mathbf{r}_{1}, \mathbf{r}_{2}\right)=D^{(1)}\left(\mathbf{r}_{1}, \mathbf{r}_{2}\right) /\left[D^{(1)}\left(\mathbf{r}_{1}, \mathbf{r}_{2}\right) D^{(1)}\left(\mathbf{r}_{2}, \mathbf{r}_{2}\right)\right]^{1 / 2} \\
& d^{(2)}\left(\mathbf{r}_{1}, \mathbf{r}_{2}\right)=D^{(2)}\left(\mathbf{r}_{1}, \mathbf{r}_{2}\right) /\left[D^{(1)}\left(\mathbf{r}_{1}, \mathbf{r}_{1}\right) D^{(1)}\left(\mathbf{r}_{2}, \mathbf{r}_{2}\right)\right]
\end{aligned}
$$

The temporal characteristics, on the other hand, are governed by the functions

$$
\begin{aligned}
c(\tau) & =\int_{-\infty}^{\infty} h_{\tau}(t) \mathrm{d} t, \\
\eta(\tau) & =\bar{h}_{0}(\tau) / \int_{-\infty}^{\infty} h_{0}^{2}(t) \mathrm{d} t, \\
\eta_{1}(\tau) & =\gamma(\tau) \circledast \bar{h}_{0}(\tau), \\
\eta_{2}(\tau) & =\int_{-\infty}^{\infty} \gamma(t) \bar{h}_{\tau}(t) \mathrm{d} t,
\end{aligned}
$$

where $h_{\tau}(t)$ and $\bar{h}_{\tau}(t)$ are related to $\xi(t)$ by

$$
\begin{aligned}
& \Gamma_{1}\left(\mathbf{r}_{1}, t_{1} ; \mathbf{r}_{2}, t_{2}\right)=\xi^{*}\left(t_{1}\right) \xi\left(t_{2}\right) D^{(1)}\left(\mathbf{r}_{1}, \mathbf{r}_{2}\right), \\
& \Gamma_{2}\left(\mathbf{r}_{1}, t_{1} ; \mathbf{r}_{2}, t_{2}\right)=\left|\xi\left(t_{1}\right)\right|^{2}\left|\xi\left(t_{2}\right)\right|^{2} D^{(2)}\left(\mathbf{r}_{1}, \mathbf{r}_{2}\right),
\end{aligned}
$$

where $D^{(1)}$ and $D^{(2)}$ are related to the spatial function $s(\mathbf{r})$ by Eqs. (3.14a) and (3.14b).

We next substitute these equations into Eqs. (3.6) and (3.7) and use the normalizations

$$
\begin{aligned}
g^{(1)}\left(\mathbf{r}_{1}, \mathbf{r}_{2}, \tau\right)= & G^{(1)}\left(\mathbf{r}_{1}, \mathbf{r}_{2}, \tau\right) / \\
& \times\left[G^{(1)}\left(\mathbf{r}_{1}, \mathbf{r}_{1}, 0\right) G^{(1)}\left(\mathbf{r}_{2}, \mathbf{r}_{2}, 0\right)\right]^{1 / 2}, \\
g^{(2)}\left(\mathbf{r}_{1}, \mathbf{r}_{2}, \tau\right)= & G^{(2)}\left(\mathbf{r}_{1}, \mathbf{r}_{2}, \tau\right) / \\
& \times\left[G^{(1)}\left(\mathbf{r}_{1}, \mathbf{r}_{1}, 0\right) G^{(1)}\left(\mathbf{r}_{2}, \mathbf{r}_{2}, 0\right)\right]
\end{aligned}
$$

to arrive at the results, which we present in the next subsection.

\section{First- and Second-Order Correlation Functions}

1. General Results

When spatial and temporal effects are separable, in accordance with Eq. (2.3), the first- and second-order correlation functions turn out to be

$$
\begin{gathered}
G^{(1)}(\mathbf{r}, \mathbf{r}, 0)=\mu \bar{n}_{0} D^{(1)}(\mathbf{r}, \mathbf{r}) \\
g^{(1)}\left(\mathbf{r}_{1}, \mathbf{r}_{2}, \tau\right)=c(\tau) d^{(1)}\left(\mathbf{r}_{1}, \mathbf{r}_{2}\right)
\end{gathered}
$$

$$
\begin{gathered}
h_{\tau}(t)=\xi^{*}(t) \xi(t+\tau), \quad h_{0}(t)=|\xi(t)|^{2}, \\
\bar{h}_{\tau}(t)=\int_{-\infty}^{\infty} h_{\tau} *\left(t^{\prime}\right) h_{\tau}\left(t^{\prime}+t\right) \mathrm{d} t^{\prime}, \\
\bar{h}_{0}(t)=\int_{-\infty}^{\infty} h_{0}\left(t^{\prime}\right) h_{0}\left(t^{\prime}+t\right) \mathrm{d} t^{\prime} .
\end{gathered}
$$

We assume, without loss of generality, that [see Eqs. (3.1) and (3.2)]

$$
\int_{-\infty}^{\infty} h_{0}(t) \mathrm{d} t=1
$$

The parameter $\delta$, which appears within the second set of brackets of $g^{(2)}$ in Eq. (3.13), is given by

$$
\delta=\mu \tau_{p},
$$

where

$$
\tau_{p}=1 / \int_{-\infty}^{\infty} h_{0}^{2}(t) \mathrm{d} t
$$
emissions per emission lifetime and acts as a degeneracy parameter. Large $\delta$ means that emission wave packets overlap which also appears within the second set of brackets of $g^{(2)}$, represents the width of the function $h_{0}(t)$, or the emission lifetime. Therefore $\delta$ represents the average number of strongly and interfere with one another. The quantity $\beta$, 
is given in Eqs. (3.3) and (3.4). Equation (3.13) is in accord with our earlier results for Poisson excitations $(\rho=0),{ }^{17}$ when $\beta=0,1,2$.

\section{Physical Interpretation}

We are now in a position to ascribe a physical interpretation to the three clusters of terms comprising $g^{(2)}\left(\mathbf{r}_{1}, \mathbf{r}_{2}, \tau\right)$, as given in Eq. (3.13). The clusters are separated by square brackets. The first, $\left[1+\left|g^{(1)}\left(\mathbf{r}_{1}, \mathbf{r}_{2}, \tau\right)\right|^{2}\right]$, is identical with that obtained for chaotic light. ${ }^{2,17}$ The second cluster is proportional to $\beta / \delta$; it is always nonnegative and represents excess bunching arising from multiple photons per emission [see Eq. (3.5)]. It vanishes under two conditions: when $\beta=0$ and/or when $\delta \gg$ 1. $\beta=0$ corresponds to the case when each emission contains one and only one photon. $\delta \gg 1$ corresponds to significant overlap of the emissions, indicating that this excess particlelike noise is washed away by substantial interference effects. The third cluster is proportional to $\rho$, which is a characteristic of the statistics of the primary excitations. For Poisson excitations, $\rho=0$. For super-Poisson excitations $\rho$ is positive, thereby adding to the bunching, whereas for sub-Poisson excitations it is negative, leading to a decrease in the bunching.

In the case of Poisson excitations, only the first two square brackets in Eq. (3.13) remain. This result is identical with that which we obtained earlier ${ }^{17}$ (although here we have also included spatial effects). It is interesting to observe that for Poisson primary excitations, and for emissions that each produce a single photon $(\beta=0), g^{(2)}=1+\left|g^{(1)}\right|^{2}$, as for chaotic light. Furthermore, as we have already pointed out, ${ }^{17}$ in the limit of dense emissions at random times, interference causes the disappearance, in the second-order correlation function, of the character of the individual emissions (arbitrary $\beta$ ). The shot-noise fluctuations are such that chaotic behavior is approached in this limit. This again points up the fundamental difficulty in attempting to generate antibunched light by Poisson excitations, as pointed out in Section 1 [see Fig. 1(a)]. On the other hand, if the excitations are sub-Poisson, then $\rho$ $<0$ and the situation shown in Fig. 1(b) can prevail. To investigate the behavior of $g^{(2)}$ in this regime, we have to make some simplifying assumptions, such as those considered below.

\section{Exponentially Decaying Wave Packets}

The temporal characteristics of the light arise from an interplay between the properties of the individuals emissions [through $\xi(t)$ ] and the properties of the renewal excitations [through $\gamma(t)$ ]. It is difficult to gain further insight into the behavior of $g^{(2)}$ in the general case without additional assumptions. Substantial progress can be made, it turns out, if we consider the individual nonstationary emissions $\xi(t)$ to be modeled as exponentially decaying sinusoids. Since this is a reasonable assumption for individual atomic emissions, we explore its consequences.

We therefore proceed to obtain the functions in Eqs. (3.16)-(3.24) in the special case when

$$
\xi(t)=\left\{\begin{array}{cl}
\left(2 / \tau_{p}\right)^{1 / 2} \exp \left(-t / \tau_{p}\right) \exp \left(i \omega_{0} t\right), & t \geq 0 \\
0, & t<0
\end{array} .\right.
$$

This leads to

$$
\begin{aligned}
h_{0}(t) & =\left\{\begin{array}{cc}
\left(2 / \tau_{p}\right) \exp \left(-2 t / \tau_{p}\right), & t \geq 0 \\
0, & t<0
\end{array}\right. \\
\bar{h}_{\tau}(t) & =\left(1 / \tau_{p}\right) \exp \left(-2|\tau| / \tau_{p}\right) \exp \left(-2|t| / \tau_{p}\right), \\
\bar{h}_{0}(t) & =\left(1 / \tau_{p}\right) \exp \left(-2|t| / \tau_{p}\right), \\
c(\tau) & =\exp \left(-|\tau| / \tau_{p}\right) \exp \left(i \omega_{0} \tau\right), \\
\eta(\tau) & =\exp \left(-2|\tau| / \tau_{p}\right)
\end{aligned}
$$

These results immediately prove useful in the next subsection.

\section{Bunching/Antibunching}

To assess the bunching/antibunching of the optical field, we consider the function $g^{(2)}(0) \equiv g^{(2)}(\mathbf{r}, \mathbf{r}, 0)$. We define bunching as $g^{(2)}(0)>1$ and antibunching as $g^{(2)}(0)<1$. From Eqs. (3.13)-(3.19),

$$
g^{(2)}(0)=2+\frac{\beta}{\delta}+2 \rho \eta_{1}(0)
$$

since, by using Eqs. (3.18) and (3.19),

$$
\eta_{1}(0)=\eta_{2}(0)=\int_{-\infty}^{\infty} \gamma(t) \bar{h}_{0}(t) \mathrm{d} t .
$$

Now, for exponentially decaying emissions, $\bar{h}_{0}(t)$ is given by Eq. (3.27b), and therefore

$$
\eta_{1}(0)=\frac{2}{\tau_{p}} \int_{0}^{\infty} \gamma(t) \exp \left(-2 t / \tau_{p}\right) \mathrm{d} t .
$$

Thus $\eta_{1}(0)$ may be computed from the Laplace transform of $\gamma(t)$. We are able to obtain explicit expressions for $g^{(2)}(0)$ for the special renewal processes considered in Part E of Appendix A. These are provided below.

1. $g^{(2)}(0)$ for Excitations with Gamma-Distributed Interevent Times

Using Eqs. (3.30), (3.32), and (A23) gives rise to

$$
\eta_{1}(0)=1-\frac{2 / \delta}{(1+2 / \mathcal{N} \delta)^{\mathcal{N}}-1},
$$

and therefore

$$
g^{(2)}(0)=\frac{\beta}{\delta}+\frac{4 / \delta}{(1+2 / \mathcal{N} \delta)^{\mathcal{N}}-1} .
$$

The behavior of this function is displayed in Fig. 5 for $\beta=0$, with $\mathcal{N}$ as a parameter. For $\mathcal{N}>1, g^{(2)}(0)$ increases gradually from 0 as $\delta$ increases, approaching a fixed value of 2 when $\delta \rightarrow$ $\infty$. Furthermore, $g^{(2)}(0)$ decreases with increasing $\mathcal{N}$ for any fixed value of $\delta$. $\mathcal{N}=1$ corresponds to Poisson primary excitations, which yields $g^{(2)}(0)=2$, independent of $\delta=\mu \tau_{p}$, in accordance with Eq. (75) of Ref. 17. Note that $\mathcal{N}$ can be between zero and unity, corresponding to bunched excitations. The result for $\mathcal{N}=0.5$ is shown in the figure. In this case, increasing interference reduces the bunching; again $g^{(2)}(0) \rightarrow$ 2 as $\delta \rightarrow \infty$.

The condition most favorable to antibunching is $\mathcal{N} \rightarrow \infty$, in which case

$$
g^{(2)}(0)=\frac{\beta}{\delta}+\frac{4 / \delta}{e^{2 / \delta}-1}
$$

For $\beta=0$, antibunching $\left[g^{(2)}(0) \leq 1\right]$ is then attained for $\delta \leq$ 1.6. 


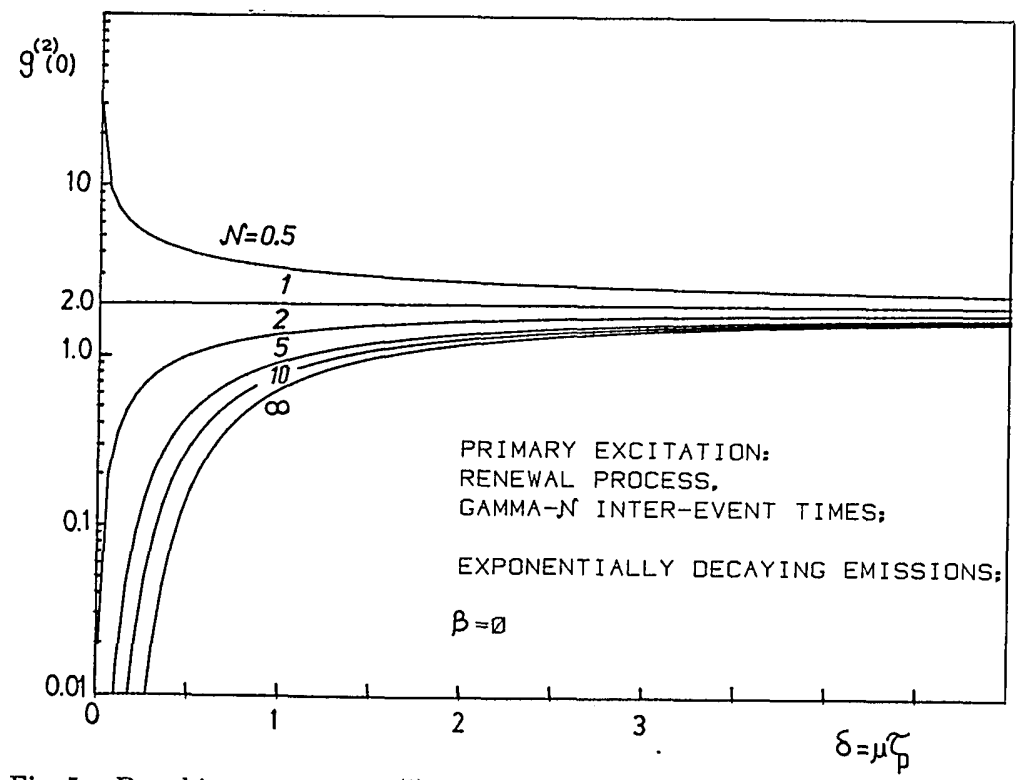

Fig. 5. Bunching parameter $g^{(2)}(0)$ versus degeneracy parameter $\delta=\mu \tau_{p}$ on a semilog scale. The excitation is assumed to be a gamma- $\mathcal{N}$ renewal process, and the emissions are taken to be exponentially decaying. It is further assumed that $\beta=0$ (single-photon emissions). In the limiting case of ideal antibunching of the excitations $(\mathcal{N} \rightarrow \infty)$, the photons will also be antibunched $\left[g^{(2)}(0)<1\right]$, provided that $\delta \leq 1.6$.

2. $g^{(2)}(0)$ for Excitations with Dead-Time-Modified

Poisson Renewal Process

With the help of Eqs. (3.30), (3.32), and (A29), we obtain

$$
\begin{aligned}
\eta_{1}(0) & =1-\frac{2 / \delta}{\left(1+\frac{2 / \delta}{1+D}\right) \exp \left(\frac{2 D / \delta}{1+D}\right)-1}, \\
g^{(2)}(0) & =\frac{\beta}{\delta}+\frac{4 / \delta}{\left(1+\frac{2 / \delta}{1+D}\right) \exp \left(\frac{2 D / \delta}{1+D}\right)-1}
\end{aligned}
$$

for the nonparalyzable dead-time-modified Poisson process. Here

$$
D=\mu \tau_{d} /\left(1-\mu \tau_{d}\right)=\lambda \tau_{d},
$$

where $\tau_{d}$ is the dead time. Equation (3.37) is presented (on a linear scale) in Fig. 6. $g^{(2)}(0)$ displays the same kind of dependence on $\delta$ as seen in Fig. 5 , with $D$ now playing the role of $\mathcal{N}$. The Poisson is recovered when $D=0$. When $D \rightarrow \infty$, results identical with those in Eq. (3.35) are obtained.

\section{3. $g^{(2)}(0)$ for Random-Phase Pulse-Train Excitations} Substituting Eq. (A.34) into Eq. (3.32), we obtain

$$
\eta_{1}(0)=1-\frac{2 / \delta}{e^{2 / \delta}-1},
$$

which leads to

$$
g^{(2)}(0)=\frac{\beta}{\delta}+\frac{4 / \delta}{e^{2 / \delta}-1} .
$$

Equation (3.40) is the same as Eq. (3.35). This is a result of the fact that the gamma interevent-time process with $\mathcal{N} \rightarrow$ $\infty$ and the nonparalyzable dead-time-modified Poisson process with $D \rightarrow \infty$ approach the ideal pulse train with random phase. In Fig. 7 we show $g^{(2)}(0)$ versus $\delta$, this time with $\beta$ as a parameter. Again, $g^{(2)}(0) \rightarrow 2$ for $\delta \gg 1$, independent of $\beta$. It is quite interesting to note that, even with perfectly antibunched excitations, it is impossible to achieve photon antibunching for $\beta \geq 0.45$.

\section{E. Temporal Coherence}

The temporal-coherence properties of the radiated field are described by the functions $g^{(1)}(\tau) \equiv g^{(1)}(\mathbf{r}, \mathbf{r}, \tau)$ and $g^{(2)}(\tau) \equiv$ $g^{(2)}(\mathbf{r}, \mathbf{r}, \tau)$. From Eqs. (3.12)-(3.19),

$$
g^{(1)}(\tau)=c(\tau)
$$

and

$$
g^{(2)}(\tau)=\left[1+|c(\tau)|^{2}\right]+\left[\frac{\beta}{\delta} \eta(\tau)\right]+\rho\left[\eta_{1}(\tau)+\eta_{2}(\tau)\right] .
$$

It is evident from Eqs. (3.16), (3.20), and (3.41) that first-order temporal coherence is ultimately determined by the function $\xi(t)$, which describes the decay of individual emissions. This quantity is independent of the statistics of the emission times, which are characterized by the function $\gamma(\tau)$.

The second-order correlation function, on the other hand, represents the two-photon counting rate and depends on both $\xi(t)$ and $\gamma(\tau)$. Equation (3.42) shows that it is composed of three clusters of terms (in square brackets). Again the first is the part that is associated with chaotic light. ${ }^{2,17}$ The second results from multiple photons in each emission. It depends on the state of the individual emissions. If the field is in a one-photon Fock state $(n=1 ; \beta=0)$, each emission contains a single photon, and the term within the second set of square brackets vanishes. The terms within the third set of square brackets are determined by the deviation of the excitation statistics from a Poisson process. They depend on both $\xi(t)$ and $\gamma(\tau)$ in an intertwined manner, as is apparent from Eqs. (3.18) and (3.19). It is difficult to assess that dependence in 


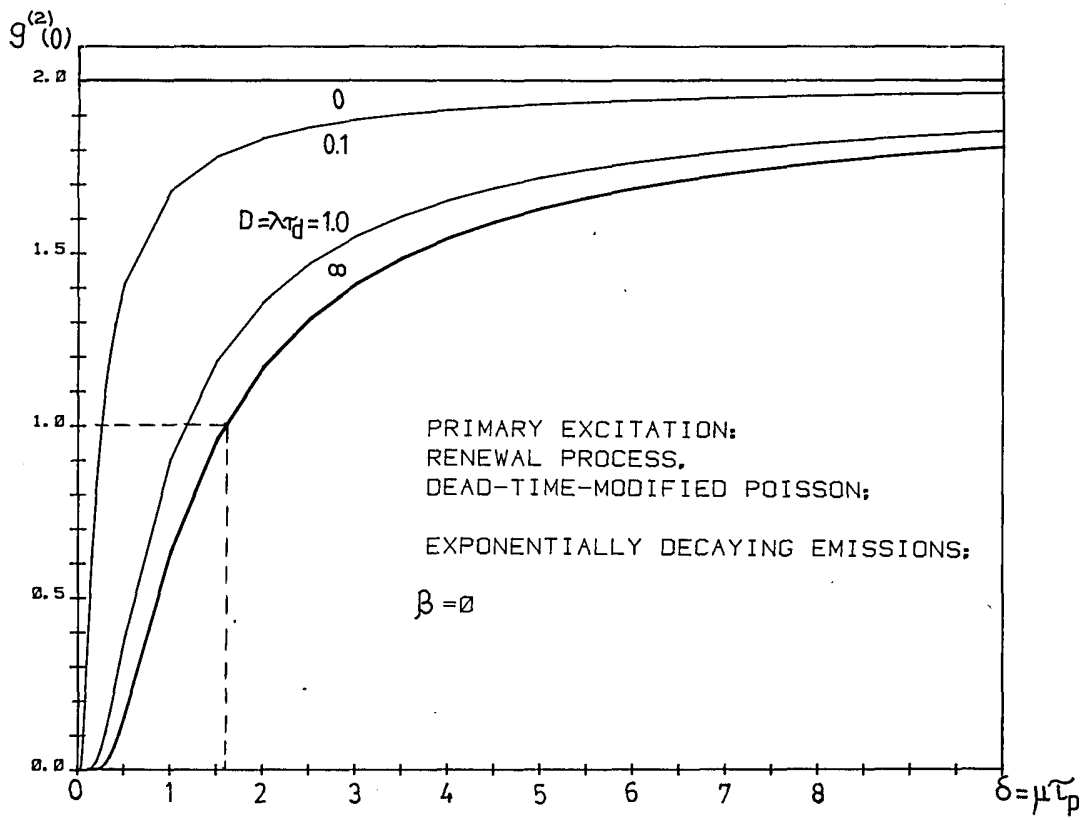

Fig. 6. Bunching parameter $g^{(2)}(0)$ versus degeneracy parameter $\delta=\mu \tau_{p}$ on a linear scale. The excitation is assumed to be a nonparalyzable dead-time-modified Poisson renewal process, and the emissions are taken to be exponentially decaying. It is further assumed that $\beta=0$ (single-photon emissions). Ideal antibunching of the excitations occurs in the limit as $D=\lambda \tau_{d} \rightarrow \infty$; this limiting curve is identical with that in Fig. 5 for $\mathcal{N} \rightarrow \infty$.

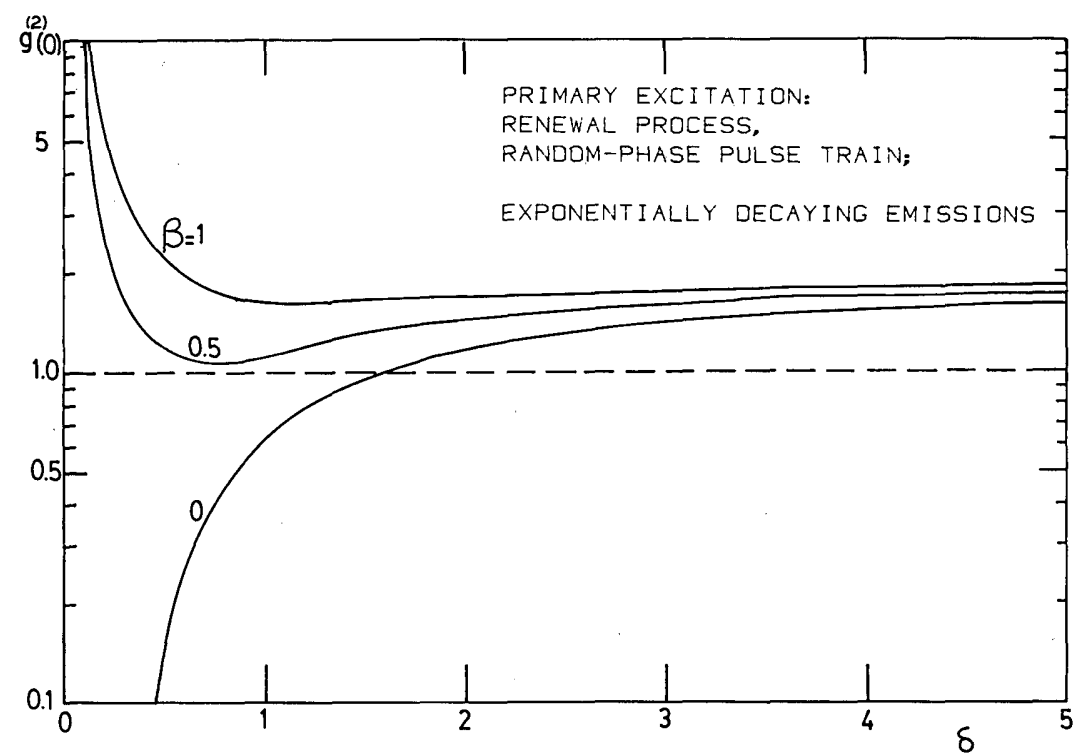

Fig. 7. Bunching parameter $g^{(2)}(0)$ versus degeneracy parameter $\delta=\mu \tau_{p}$ on a semilog scale. The excitation is assumed to be a random-phase pulse train, and the emissions are taken to be exponentially decaying. The curve for $\beta=0$ is identical with that displayed in Fig. $5(\mathcal{N}=\infty)$ and Fig. $6(D=\infty)$, demonstrating that the photons will be antibunched for $\delta \leq 1.6$. It is clear from the figure that it is impossible to achieve antibunching for coherent-state individual emissions $(\beta=1)$ or even for two-photon individual emissions. It turns out that $g^{(2)}(0)$ can be made $<1$ only for $\beta<0.45$.

general. In the following, therefore, we evaluate $g^{(2)}(\tau)$ in some interesting special cases and in some useful limits.

\section{1. $g^{(2)}(\tau)$ for Gamma-2 Excitations and Exponentially} Decaying Emissions

When the emission times follow a gamma-2 renewal process, $\gamma(\tau)$ is exponential, as in Eq. (A22). If, furthermore, the time decay of each emission is governed by an exponentially decaying function $\xi(t)$, as in Eq. (3.25), the quantities in Eq. (3.42) may be evaluated. The final result is

$$
\begin{aligned}
g^{(2)}(\tau) & =\left[1+\exp \left(-2 \tau / \tau_{p}\right)\right]+\left[\frac{\beta}{\delta} \exp \left(-2 \tau / \tau_{p}\right)\right] \\
& -\left(1-4 \delta^{2}\right)^{-1}\left[\exp \left(-4 \delta \tau / \tau_{p}\right)+(1-4 \delta) \exp \left(-2 \tau / \tau_{p}\right)\right] .
\end{aligned}
$$




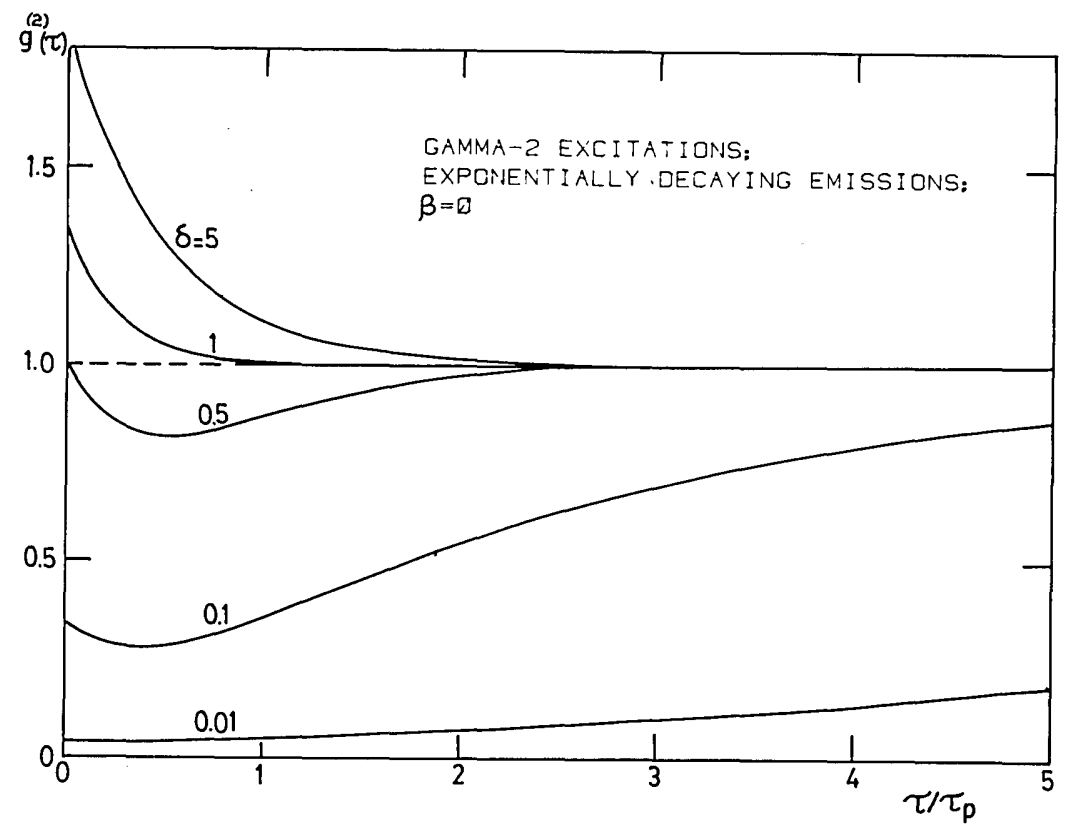

Fig. 8. Temporal coherence illustrated by the second-order normalized correlation function $g^{(2)}(\tau)$ versus $\tau$ on a linear scale. Curves are shown for various values of the degeneracy parameter $\delta=\mu \tau_{p}$. The excitations are assumed to be drawn from a gamma-2 renewal process, and the emissions are taken to be exponentially decaying. It is further assumed that $\beta=0$ (single-photon emissions), although as $\delta \rightarrow \infty$ the results are independent of $\beta$.

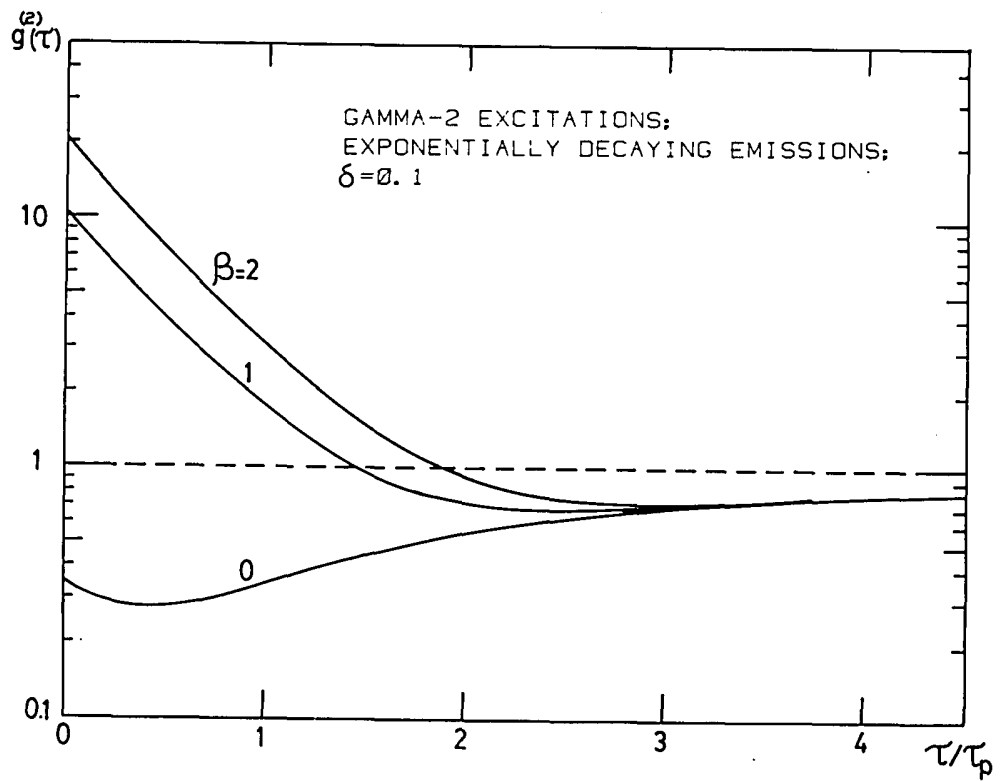

Fig. 9. Temporal coherence illustrated by the second-order normalized correlation function $g^{(2)}(\tau)$ versus $\tau$ on a semilog scale. Curves are shown for various values of $\beta$ : $n=1$ Fock state emissions $(\beta=0)$; quasi-coherent emissions $(\beta=1)$; chaotic emissions $(\beta=2)$. The degeneracy parameter $(\delta=0.1)$ is chosen to be low. The excitations are assumed to be drawn from a gamma-2 renewal process, and the emissions are taken to be exponentially decaying.

This function is shown in Fig. 8 for $\beta=0$ with $\delta$ as a parameter and in Fig. 9 for $\delta=0.1$ with $\beta$ as a parameter. Figure 8 demonstrates that the character of $g^{(2)}(\tau)$ changes dramatically with $\delta$. For small $\delta, g^{(2)}(\tau)$ is a nonmonotonic function of $\tau$ whose value always remains below unity. The light is antibunched $\left[g^{(2)}(0)<1\right]$. For large $\delta, g^{(2)}(\tau)$ is an exponentially decreasing function of $\tau$ whose value always remains above unity. The light is bunched. For much larger $\delta$, the ensuing interference is so pervasive that the results for chaotic light are recovered, independent of $\beta$. To point up the dependence of $g^{(2)}(\tau)$ on $\beta$, we must therefore go to a low value of $\delta$, as illustrated in Fig. 9, where $\delta$ is chosen to be 0.1 . Increasing $\beta$ is seen to increase the bunching because of multiple emissions that increase the coincidence rate. But 
note that all curves approach one another (at a value below unity) for $\tau / \tau_{p} \gtrsim 3$, which corresponds to $\mu \tau \gtrsim 3 \delta$ or to $\mu \tau \gtrsim$ 0.3 in this example. It is important to observe that $g^{(2)}(\tau)$ can dip below unity, even for highly clustered chaotic emissions. The key issue is the relative time scales of the excitations and the emissions.

\section{2. $g^{(2)}(\tau)$ for Pulse-Train Excitations and Exponentially}

Decaying Emissions

When the excitation is a pulse train of random phase, the function $\gamma(t)$ is given by Eq. (A34). Therefore, using Eqs. (3.18) and (3.19), we obtain (for the case of arbitrary emissions)

$$
\begin{aligned}
& \eta_{1}(\tau)=1-T_{0} \sum_{l \neq 0} \bar{h}_{0}\left(\tau-l T_{0}\right), \\
& \eta_{2}(\tau)=|c(\tau)|^{2}-T_{0} \sum_{l \neq 0} \bar{h}_{\tau}\left(\tau-l T_{0}\right) .
\end{aligned}
$$

The quantity $T_{0}=1 / \mu$ is the periodicity of the pulse train. Substituting these results in Eq. (3.42), and noting that $\rho=$ -1 , we obtain

$$
g^{(2)}(\tau)=\frac{\beta}{\delta} \eta(\tau)+T_{0} \sum_{l \neq 0} \bar{h}_{0}\left(\tau-l T_{0}\right)+T_{0} \sum_{l \neq 0} \bar{h}_{\tau}\left(\tau-l T_{0}\right) .
$$

For the particular case of exponentially decaying emissions, we use Eqs. (3.25)-(3.29) to arrive at

$$
\begin{aligned}
g^{(2)}(\tau)= & \frac{\beta}{\delta} \exp \left(-2 \tau / \tau_{p}\right)+\left[1+\exp \left(-2 \tau / \tau_{p}\right)\right] \frac{1}{\delta} \\
& \times \sum_{l \neq 0} \exp \left(-2\left|\tau-l T_{0}\right| / \tau_{p}\right) .
\end{aligned}
$$

Equation (3.47) may be rewritten in the form

$$
\begin{aligned}
& g^{(2)}(\tau)=\frac{\beta}{\delta} \exp \left(-2 \tau / \tau_{p}\right)+\frac{1}{\delta}\left[1+\exp \left(-2 \tau / \tau_{p}\right)\right] \\
& \quad \times\left[\frac{\exp (-2 \theta / \delta)+\exp [-2(1-\theta) / \delta]}{1-\exp (-2 / \delta)}-\exp \left(-2 \tau / \tau_{p}\right)\right],
\end{aligned}
$$

where $\theta=\theta(\tau)$ is a periodic triangular function of period $T_{0}$, i.e.,

$$
\begin{array}{ll}
\theta(\tau)=\tau / T_{0}, & 0 \leq \tau<T_{0}, \\
\theta\left(\tau+l T_{0}\right)=\theta(\tau), & l=1,2, \ldots
\end{array}
$$

For the special region $\tau \leq T_{0}, \theta=\tau / T_{0}, \theta / \delta=\tau / \tau_{p}$, and

$$
\begin{aligned}
g^{(2)}(\tau)= & \frac{\beta}{\delta} \exp \left(-2 \tau / \tau_{p}\right)+\frac{2 / \delta}{e^{2 / \delta}-1} \\
& \times\left[1+\exp \left(-2 \tau / \tau_{p}\right)\right] \cosh \left(2 \tau / \tau_{p}\right), \quad \tau \leq T_{0} .
\end{aligned}
$$

For $\tau=0$, Eq. (3.35) is recovered.

In the limit of small $\delta, \mathrm{Eq}$. (3.48) gives rise to

$$
g^{(2)}(\tau)=\beta T_{0} \delta(\tau)+T_{0} \sum_{l \neq 0}[1+\exp (-2 l / \delta)] \delta\left(\tau-l T_{0}\right)
$$

This is a train of $\delta$ functions spaced at multiples of $T_{0}$ along the $\tau$ axis. For $\beta=0, g^{(2)}(0)=0$, confirming that the twophoton coincidence rate is zero. We also consider the limit of large $\delta$; Eq. (3.50) gives the (special-region) result that

$$
\begin{gathered}
g^{(2)}(\tau)=\left[1+\exp \left(-2 \tau / \tau_{p}\right)\right] \cosh \left(2 \tau / \tau_{p}\right) \\
\delta \gg 1, \quad \tau \leq T_{0}
\end{gathered}
$$

independent of $\beta$. This function has a value of 2 at $\tau=0 ; \mathrm{Eq}$. (3.48) shows that $g^{(2)}(\tau)$ settles at unity as $\tau \rightarrow \infty$ for large $\delta$.

In Fig. 10 we display the dependence of $g^{(2)}(\tau)$ on $\tau / T_{0}$ for $\beta=0$, with $\delta$ as a parameter. For pulse-train excitations, $\mu$ $=1 / T_{0}$, so that $\delta=\mu \tau_{p}=\tau_{p} / T_{0}$. Thus low $\delta$ means that single photons are emitted at well-spaced regular intervals, with little chance of interference between the wave packets. The two-photon counting rate $g^{(2)}(\tau)$ is 0 at $\tau=0$ but becomes large at separations that are in the vicinity of integer multiples of $T_{0}$. As $\delta$ becomes large, interference becomes increasingly important and there is a gradual shift toward the classical behavior of $g^{(2)}(\tau)$. For $\delta \gg 1, g^{(2)}(0)$ begins at 2 and decays exponentially to unity as $\tau \rightarrow \infty$. The behavior of $g^{(2)}(\tau)$ in this region would be more familiar in a graphical representation in which the abscissa is $\tau / \tau_{p}$ rather than $\tau / T_{0}$.

3. $g^{(2)}(\tau)$ for Instantaneous Emissions $\left(\tau_{p} \ll \tau_{e}\right.$ or $\left.\delta \ll 1\right)$ We have been able to obtain a number of results for $g^{(2)}(\tau)$ under the assumption of exponentially decaying emissions. We now take a different approach in which we examine some limiting results for $g^{(2)}(\tau)$ without making specific assumptions about the form of $\bar{h}_{0}(t)$ and $\gamma(\tau)$.

If $\tau_{p}$, the width of the functions $\xi(t), h_{0}(t)$, and $\bar{h}_{0}(t)$, is much smaller than $\tau_{e}$, the width of the function $\gamma(\tau)$, then Eqs. (3.18) and (3.19) may be approximated by

$$
\eta_{1}(\tau) \approx \gamma(\tau) \int_{-\infty}^{\infty} \bar{h}_{0}(t) \mathrm{d} t=\gamma(\tau)
$$

PULSE-TRAIN EXCITATIONS; EXPONENTIALLY DECAYING EMISSIONS;

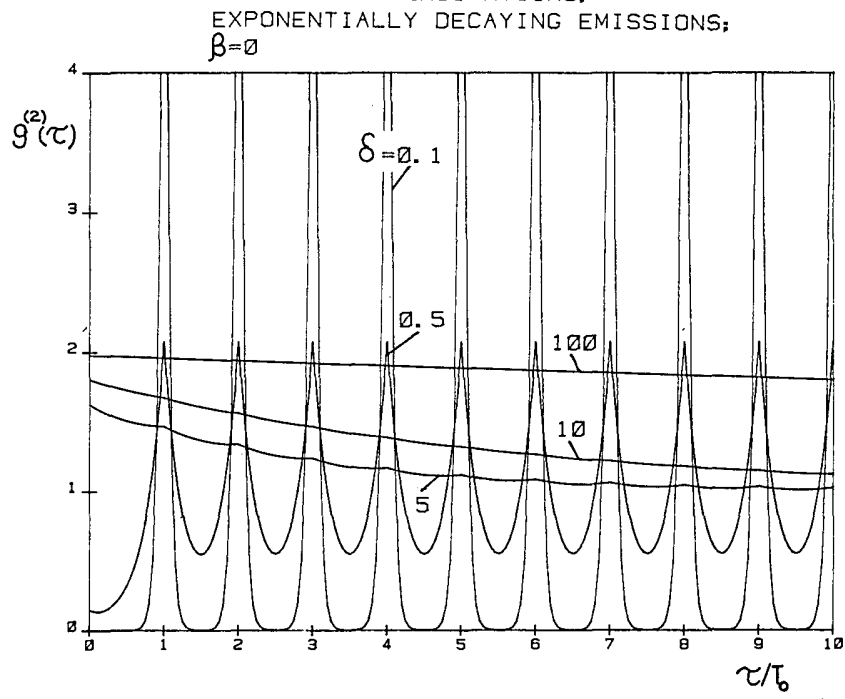

Fig. 10. Temporal coherence illustrated by the second-order normalized correlation function $g^{(2)}(\tau)$ versus $\tau / T_{0}$ on a linear scale. Curves are shown for various values of the degeneracy parameter $\delta$ $=\mu \tau_{p}=\tau_{p} / T_{0}$. Pulse-train excitations are assumed, and the emissions are taken to be exponentially decaying. It is assumed that $\beta$ $=0$ (single-photon emissions), although as $\delta \rightarrow \infty$ the results are independent of $\beta$. 

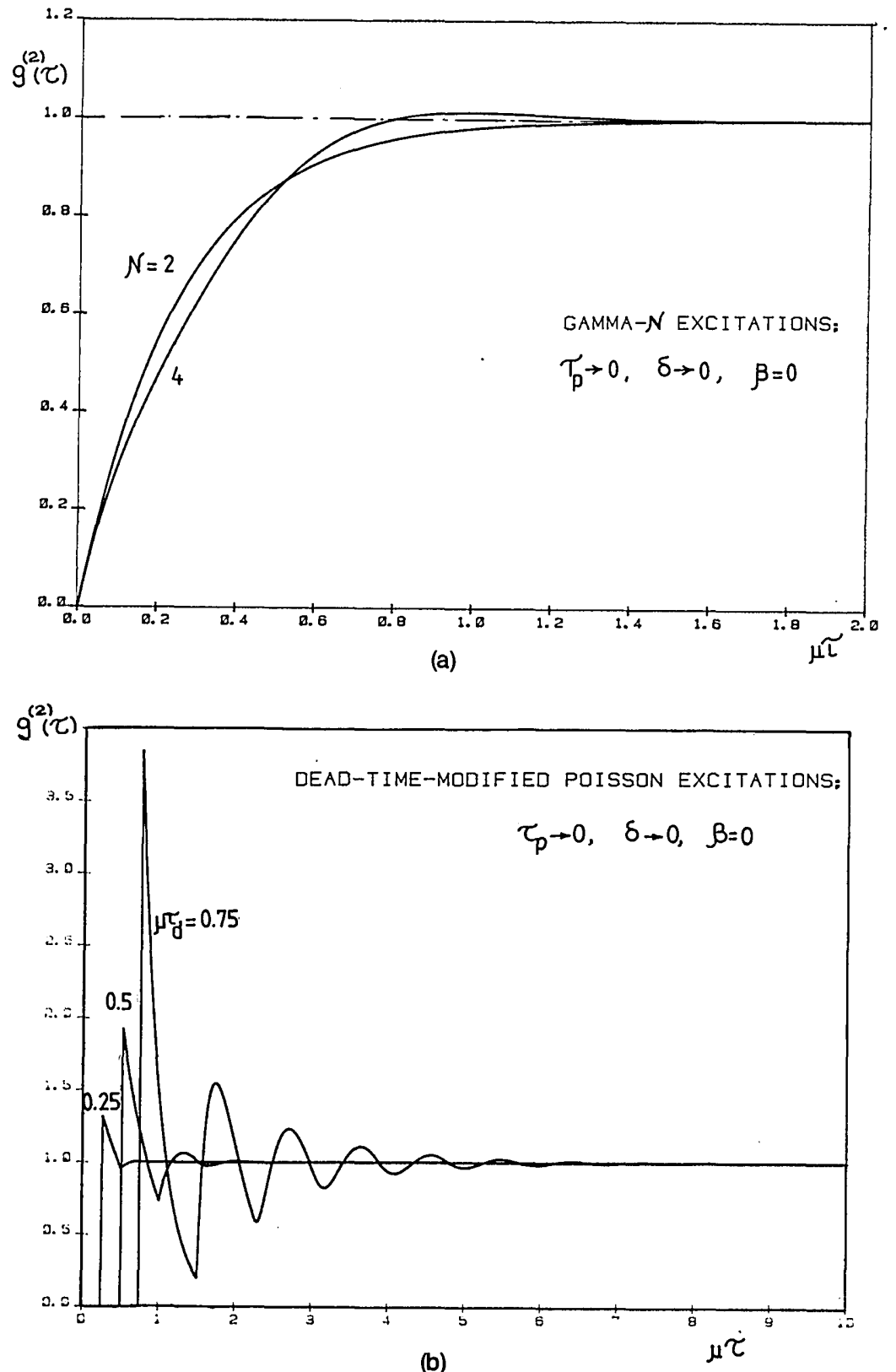

Fig. 11. Second-order normalized correlation function $g^{(2)}(\tau)$ versus $\mu \tau$. These results apply for instantaneous single-photon emissions, i.e., when $\tau_{p} \ll \tau_{e}$ (or $\delta \ll$ 1) and $\beta=0$. (a) Gamma-2 and gamma-4 excitations; (b) dead-time-modified Poisson excitations.

$$
\eta_{2}(\tau) \approx \int_{-\infty}^{\infty} \bar{h}_{\tau}(t) \mathrm{d} t=|c(\tau)|^{2}
$$

In this limiting case, the emission lifetime is much shorter than the time over which the primary excitations are correlated, i.e., the emissions are instantaneous. We may convert this condition $\left(\mu \tau_{p} \ll \mu \tau_{e}\right)$ to a condition on the degeneracy parameter in a rather rough manner. For gamma- $\mathcal{N}$ excitations, $\tau_{e} \sim 1 / \mu$, whereas for dead-time-modified Poisson excitations, $\mu \tau_{e} \sim \mu \tau_{d}\left(2-\mu \tau_{d}\right) \sim \mu \tau_{d} \lesssim 1$ (see Appendix A). This leads us to the equivalent condition $\mu \tau_{p}=\delta \ll 1$. The assumption of instantaneous emissions is essentially equivalent to the assumption of the absence of interference between emissions, as is intuitively plausible. Equation (3.42) then becomes

$$
g^{(2)}(\tau) \approx\left[1+|c(\tau)|^{2}\right]+\left[\frac{\beta}{\delta} \eta(\tau)\right]+\rho\left[\gamma(\tau)+|c(\tau)|^{2}\right]
$$

In all the renewal-process examples considered in Appendix A, $\rho=-1$ (except for the Poisson, where $\rho=0$ ). The second terms within the first and third sets of brackets therefore cancel, resulting in the simplified formula

$$
g^{(2)}(\tau) \approx 1-\gamma(\tau)+\frac{\beta}{\delta} \eta(\tau)
$$

Since this expression is valid only for $\delta \ll 1$, multiple-photon emissions will contribute substantially to $g^{(2)}(\tau)$. 
Considering the special case of one-photon emissions, for which $\beta=0$, we obtain

$$
g^{(2)}(\tau) \approx 1-\gamma(\tau)
$$

The quantities $g^{(2)}(\tau)$ and $\gamma(\tau)$ differ by a constant since the former is a correlation function whereas the latter is a covariance function. Under these conditions, the second-order temporal correlation function for the light is determined entirely by the correlation function of the primary excitations. This is an important result. It tells us that the correlation properties of the excitations are directly transferred to the correlation properties of the photons. In particular, if the excitations are antibunched [e.g., if $\rho \gamma(0)=-1, \gamma(0)=1$ ], then the photons will also be antibunched $\left[g^{(2)}(0)=0\right]$. The important issue in producing antibunching is to avoid interference, which destroys it.

This behavior is illustrated graphically in Fig. 11, where we present $g^{(2)}(\tau)$ versus $\mu \tau$, calculated from expression (3.57). This expression is applicable when $\tau_{p} \ll \tau_{e}$ (or $\left.\delta \ll 1\right)$ and $\beta$ $=0$. In Fig. 11(a) we show the result for gamma-2 and gamma-4 primary excitations. In Fig. 11(b) we show the result for dead-time-modified Poisson primary excitations, for several values of the parameter $\mu \tau_{d}$. At a value of $\mu \tau_{d}=0.75$, the correlation function begins to show features that resemble the correlation function shown in Fig. 10 (for $\delta=0.1$ ). In the limit $\mu \tau_{d} \rightarrow 1$, the dead-time-modified Poisson correlation function should become identical with the pulse-train correlation function with $\delta \rightarrow 0$.

The results for Poisson excitations require a special word. The covariance function in this case is a $\delta$ function, so that the condition of validity for this section, namely, that the width of functions such as $\bar{h}_{0}(t)$ be less than the width of $\gamma(\tau)$, cannot be satisfied. Thus formulas (3.56) and (3.57) are not applicable for Poisson excitations.

\section{4. $g^{(2)}(\tau)$ for Dense Emissions $\left(\tau_{p} \gg \tau_{\mathrm{e}}\right.$ or $\left.\delta \gg 1\right)$}

We now consider the opposite limit in which $\tau_{p} \gg \tau_{e}$. For gamma- $\mathcal{N}$ excitations $\tau_{e} \sim 1 / \mu$, whereas for dead-timemodified Poisson excitations $\mu \tau_{e} \sim \mu \tau_{d} \lesssim 1$. Thus $\mu \tau_{p} \gg$ $\mu \tau_{e}$ is essentially equivalent to $\delta \gg 1$. Interference therefore plays an important role. Returning to Eqs. (3.18) and (3.19), we may approximate them as

$$
\begin{aligned}
& \eta_{1}(\tau) \approx \tau_{e} \bar{h}_{0}(\tau), \\
& \eta_{2}(\tau) \approx \tau_{e} \bar{h}_{\tau}(0)=\tau_{e} \bar{h}_{0}(\tau)
\end{aligned}
$$

in this limit. From Eqs. (3.17) and (3.24),

$$
\eta_{1}(\tau) \approx \eta_{2}(\tau) \approx\left(\tau_{e} / \tau_{p}\right) \eta(\tau),
$$

so that Eq. (3.42) becomes

$$
g^{(2)}(\tau) \approx\left[1+|c(\tau)|^{2}\right]+\frac{\beta}{\delta} \eta(\tau)+2 \rho\left(\tau_{e} / \tau_{p}\right) \eta(\tau)
$$

Because $\tau_{p} \gg \tau_{e}$, and $\eta(\tau)$ is normalized such that its value is always less than unity, the last term in expression (3.61) may be neglected, leaving

$$
g^{(2)}(\tau) \approx 1+|c(\tau)|^{2}+\frac{\beta}{\delta} \eta(\tau)
$$

A further simplification occurs since $\delta \gg 1$, whereupon we obtain the final result that

$$
g^{(2)} \tau \approx 1+|c(\tau)|^{2}
$$

Expression (3.63) is identically the second-order correlation function for chaotic light, independently of $\rho$ and $\gamma(\tau)$ (the excitation statistics) and of $\beta$ (the state of the individual emissions).

This is understood in terms of the following. In the limit of dense emissions at random times, many emissions add together randomly, producing a stationary circularly complex Gaussian process for the optical field, in accordance with the central-limit theorem. This, in turn, leads to the result for chaotic light represented in expression (3.63). The outcome is the same whatever the nature of the excitations and the individual emissions. Only one characteristic of the latter survives; it is the spectrum of the emissions.

This behavior has already been seen earlier: In Figs. 5-7, $g^{(2)}(0)$ has been shown to approach the value 2 as $\delta \rightarrow \infty$; in Fig. $8, g^{(2)}(\tau)$ approaches expression (3.63) for $\delta=5$; and in Fig. $10, g^{(2)}(\tau)$ approaches expression (3.63) for $\delta=100$.

With regard to Poisson excitations, the $\delta$-function covariance causes expression (3.62) to be obeyed for arbitrary degeneracy parameter, as we know from our earlier work. ${ }^{17}$ Of course when $\delta \gg 1$, we recover expression (3.63).

\section{F. Spatial Coherence}

The spatial-coherence properties of the radiated field, in the first and second order, are expressed by the functions $g^{(1)}\left(\mathbf{r}_{1}\right.$, $\left.\mathbf{r}_{2}, 0\right)$ and $g^{(2)}\left(\mathbf{r}_{1}, \mathbf{r}_{2}, 0\right)$ in Eqs. (3.12) and (3.13). These relations, in turn, are determined by the functions $s(\mathbf{r})$ and $d^{(1)}\left(\mathbf{r}_{1}\right.$, $\left.\mathbf{r}_{2}\right), d^{(2)}\left(\mathbf{r}_{1}, \mathbf{r}_{2}\right)$ in Eqs. (3.14) and (3.15). $s(\mathbf{r})$ describes the radiation from a point in the source to the detector. In the quasi-monochromatic approximation, ${ }^{26}$ this function is the spherical wave

$$
s(\mathbf{r})=e^{-i k r} / r,
$$

where $k$ and $r$ represent the magnitudes of the wave vector of the light and the distance between a point in the detector and a point in the source, respectively. Using Eq. (3.64), we obtain

$$
D^{(1)}\left(\mathbf{r}_{1}, \mathbf{r}_{2}\right)=\frac{1}{V} \int_{V} \frac{\exp \left[-i k\left(\left|\mathbf{r}_{2}-\mathbf{r}^{\prime}\right|-\left|\mathbf{r}_{1}-\mathbf{r}^{\prime}\right|\right)\right] \mathrm{d} \mathbf{r}^{\prime}}{\left|\mathbf{r}_{2}-\mathbf{r}^{\prime}\right|\left|\mathbf{r}_{1}-\mathbf{r}^{\prime}\right|}
$$

and, in the paraxial approximation, ${ }^{26}$

$$
d^{(2)}\left(\mathbf{r}_{1} \mathbf{r}_{2}\right)=1 \text {. }
$$

Substituting these results into Eqs. (3.12) and (3.13), we obtain expressions for $g^{(1)}\left(\mathbf{r}_{1}, \mathbf{r}_{2}\right) \equiv g^{(1)}\left(\mathbf{r}_{1}, \mathbf{r}_{2}, 0\right)$ and $g^{(2)}\left(\mathbf{r}_{1}, \mathbf{r}_{2}\right)$ $\equiv g^{(2)}\left(\mathbf{r}_{1}, \mathbf{r}_{2}, 0\right)$ :

$g^{(1)}\left(\mathbf{r}_{1}, \mathbf{r}_{2}\right)=d^{(1)}\left(\mathbf{r}_{1}, \mathbf{r}_{2}\right)$,

$g^{(2)}\left(\mathbf{r}_{1}, \mathbf{r}_{2}\right)=\left[1+\rho \eta_{1}(0)\right]\left[1+\left|g^{(1)}\left(\mathbf{r}_{1}, \mathbf{r}_{2}\right)\right|^{2}\right]+\beta / \delta$.

By using Eq. (3.30), this may be written in the form

$$
g^{(2)}\left(\mathbf{r}_{1}, \mathbf{r}_{2}\right)=\frac{1}{2}\left[g^{(2)}(0)-\beta / \delta\right]\left[1+\left|g^{(1)}\left(\mathbf{r}_{1}, \mathbf{r}_{2}\right)\right|^{2}\right]+\beta / \delta,
$$

thereby relating the second-order spatial-coherence properties to the bunching/antibunching properties of the light. 
Equations (3.15a), (3.65), and (3.67) give rise to a first-order spatial-coherence function that is identical with that for a completely incoherent source of uniform intensity, as obtained from the van Cittert-Zernike theorem. ${ }^{26}$ Our source is therefore totally equivalent, in its first-order spatial behavior, to the classical result.

In the second order, however, our source exhibits properties that are, in general, different from those of an ordinary chaotic source. ${ }^{26,27}$ In certain circumstances, however, the distinction vanishes and we recover the classical result for chaotic light, viz.,

$$
g^{(2)}\left(\mathbf{r}_{1}, \mathbf{r}_{2}\right) \approx 1+\left|g^{(1)}\left(\mathbf{r}_{1}, \mathbf{r}_{2}\right)\right|^{2} .
$$

It is easy to see that expression (3.70) arises from Eq. (3.68) under the following conditions:

(1) If $\beta=0$ (each emission consists of a single photon) and $\rho=0$ (the emission times follow a Poisson point process), expression (3.70) follows from Eq. (3.68).

(2) If the degeneracy parameter $\delta=\mu \tau_{p}$ is sufficiently large, then $\beta / \delta \ll 1$ and $\eta_{1}(0) \ll 1$, whereupon expression (3.70) again follows from Eq. (3.68).

If, on the other hand, the only condition that we impose is that $\beta=0$, then from Eq. (3.69) we find that

$$
g^{(2)}\left(\mathbf{r}, \mathbf{r}_{2}\right)=\frac{1}{2} g^{(2)}(0)\left[1+\left|g^{(1)}\left(\mathbf{r}_{1}, \mathbf{r}_{2}\right)\right|^{2}\right] .
$$

The bunching parameter $g^{(2)}(0)$ has been studied in depth in Section 3. For sub-Poisson primary excitations with $\beta=0$, $g^{(2)}(0)$ generally increases monotonically from 0 to 2 as $\delta$ increases (see Figs. 5, 6, 8, and 10). The two-photon counting rate is therefore seen to exhibit precisely the same spatial dependence as it does for chaotic light, but with a magnitude that can be substantially reduced. In particular, when $g^{(2)}(0)$ $=0$, the photon emissions are spaced apart in time so that the two-photon counting rate is zero for all points on the receiving plane, as is evident from Eq. (3.71).

As an example, we consider Young's experiment. Imagine a source composed of two incremental volumes (points) at positions $\left(x^{\prime}=0, y^{\prime}=a / 2, z^{\prime}=0\right)$ and $\left(x^{\prime}=0, y^{\prime}=-a / 2, z^{\prime}=\right.$ $0)$. The observation points $\mathbf{r}_{1}$ and $\mathbf{r}_{2}$ are located in a plane perpendicular to the $z$ axis, a distance $z$ away. Assuming that $z$ is sufficiently large for the Fraunhofer approximation to be applicable, ${ }^{26}$ it can be shown from Eqs. (3.15a), (3.65), (3.67), and (3.68) that

$$
\begin{aligned}
g^{(1)}\left(\mathbf{r}_{1}, \mathbf{r}_{2}\right) & =\cos \left[\frac{\pi}{L}\left(y_{1}-y_{2}\right)\right], \\
g^{(2)}\left(\mathbf{r}_{1}, \mathbf{r}_{2}\right) & =\frac{1}{2}\left[g^{(2)}(0)-\beta / \delta\right]\left[1+\cos ^{2} \frac{\pi}{L}\left(y_{1}-y_{2}\right)\right]+\beta / \delta \\
& =\frac{3}{4}\left[g^{(2)}(0)-\beta / \delta\right]\left[1+\frac{1}{3} \cos \frac{2 \pi}{L}\left(y_{1}-y_{2}\right)\right]+\beta / \delta .
\end{aligned}
$$

Here $L=\lambda z / a$, where $\lambda$ is the wavelength of the light. The joint probability of finding one photon at $y_{1}$ and one photon at $y_{2}$ depends on $\left(y_{1}-y_{2}\right)$ in a sinusoidal fashion.

The two-photon fringe pattern has a visibility $\mathcal{V}^{(2)}$ given by

$$
\mathcal{V}^{(2)}=\left|\frac{g^{(2)}(0)-\beta / \delta}{3 g^{(2)}(0)+\beta / \delta}\right| .
$$

For $\beta=0$ and/or $\delta \rightarrow \infty, \mathcal{V}^{(2)}=1 / 3$ (as for chaotic light 26,27 ), independent of the primary excitation statistics. As $\beta$ increases, however, $\mathcal{V}^{(2)}$ decreases. Our model (and our result) differs somewhat from that recently dealt with by Mandel. ${ }^{27}$ $\mathrm{He}$ considered the spatial-coherence properties for Young's experiment in which each source point is composed of a fixed (or random) number of atomic emitters, in the absence of time dependence.

\section{PHOTON-COUNT STATISTICS}

Having studied the coherence properties (correlation functions) of the light generated by our model, we turn now to an investigation of the photon-count statistics. Let $n$ be the number of photons that cross an area $A$ of the detector during the time interval $[t, t+T]$. Because of stationarity, the statistics of $n$ are independent of $t$. We first determine the mean and the variance of $n$, and then we discuss the counting probability distribution under some limiting conditions.

\section{A. Count Mean and Variance}

These quantities are determined from the first- and secondorder correlation functions, respectively. The mean number of counts $\langle n\rangle$ is given by the integral of the one-photoncounting rate, i.e., ${ }^{2,3,28}$

$$
\langle n\rangle=\int_{A} \int_{0}^{T} G^{(1)}(\mathbf{r}, \mathbf{r}, t) \mathrm{d} t \mathrm{~d} \mathbf{r} .
$$

Using Eq. (3.11), we obtain

$$
\langle n\rangle=\mu T\langle x\rangle,
$$

where

$$
\langle x\rangle=\bar{n}_{0} \int_{A} D^{(1)}(\mathbf{r}, \mathbf{r}) \mathrm{d} \mathbf{r} .
$$

Here $\mu T$ is the average number of emissions in the countingtime interval $T, \bar{n}_{0}$ is the average number of photons per emission, and $\langle x\rangle$ is the average number of photons per emission registered by the detector. The integral in Eq. (4.3) represents the collection efficiency of a detector of area $A$; also included in this quantity is the internal efficiency of the detector itself.

The variance of the count number, $\operatorname{Var}(n)$, is related to the second-order correlation function $g^{(2)}\left(\mathbf{r}_{1}, \mathbf{r}_{2}, t\right)$ by ${ }^{2,3,29}$

$$
\begin{aligned}
\operatorname{Var}(n)= & \langle n\rangle+\langle n\rangle^{2} \frac{1}{A^{2}} \frac{1}{T^{2}} \int_{0}^{T} \int_{0}^{T} \iint_{A} \\
& \times\left[g^{(2)}\left(\mathbf{r}_{1}, \mathbf{r}_{2}, t_{2}-t_{1}\right)-1\right] \mathrm{d} \mathbf{r}_{1} \mathrm{~d} \mathbf{r}_{2} \mathrm{~d} t_{1} \mathrm{~d} t_{2} .
\end{aligned}
$$

Using Eqs. (3.12), (3.13), and (3.66), we obtain

$$
\begin{aligned}
\operatorname{Var}(n)= & \langle n\rangle+\langle n\rangle^{2} \frac{1}{A^{2}} \frac{2}{T} \int_{0}^{T} \iint_{A}\left(1-\frac{\tau}{T}\right) \\
& \times\left\{\left|c(\tau) d^{(1)}\left(\mathbf{r}_{1}, \mathbf{r}_{2}\right)\right|^{2}+\frac{\beta}{\mu \tau_{p}} \eta(\tau)\right. \\
& \left.+\rho\left[\eta_{1}(\tau)+\eta_{2}(\tau)\left|d^{(1)}\left(\mathbf{r}_{1}, \mathbf{r}_{2}\right)\right|^{2}\right]\right\} \mathrm{d} \mathbf{r}_{1} \mathrm{~d} \mathbf{r}_{2} \mathrm{~d} \tau,
\end{aligned}
$$

where we have taken $d^{(2)}\left(\mathbf{r}_{1}, \mathbf{r}_{2}\right)=1$. This then leads to 
$\operatorname{Var}(n)=\left[\langle n\rangle+\frac{\langle n\rangle^{2}}{M M_{s}}\right]+\left[\frac{\beta\langle x\rangle}{\mathcal{M}}\langle n\rangle\right]+\rho\left[\frac{\langle n\rangle^{2}}{M_{1}}+\frac{\langle n\rangle^{2}}{M_{2} M_{s}}\right]$

where

$$
\begin{aligned}
M_{s}^{-1} & =\frac{1}{A^{2}} \iint_{A}\left|d^{(1)}\left(\mathbf{r}_{1}, \mathbf{r}_{2}\right)\right|^{2} \mathrm{~d} \mathbf{r}_{1} \mathrm{~d} \mathbf{r}_{2} \\
M^{-1} & =\frac{2}{T} \int_{0}^{T}(1-\tau / T)|c(\tau)|^{2} \mathrm{~d} \tau \\
M_{1}-1 & =\frac{2}{T} \int_{0}^{T}(1-\tau / T) \eta_{1}(\tau) \mathrm{d} \tau \\
M_{2}^{-1} & =\frac{2}{T} \int_{0}^{T}(1-\tau / T) \eta_{2}(\tau) \mathrm{d} \tau \\
M^{-1} & =\frac{2}{\tau_{p}} \int_{0}^{T}(1-\tau / T) \eta(\tau) \mathrm{d} \tau
\end{aligned}
$$

The parameter $M_{s}$ represents the spatial degrees of freedom, ${ }^{28,29}$ whereas the quantities $M, M_{1}, M_{2}$, and $M$ represent temporal degrees of freedom associated with the functions $|c(\tau)|^{2}, \eta_{1}(\tau), \eta_{2}(\tau)$, and $\eta(\tau)$, respectively.

The expression for the count variance is composed of three distinct contributions, indicated by the square brackets in Eq. (4.6). These are obviously directly associated with the three contributions comprising the second-order correlation function in Eq. (3.13). The first cluster is identically the variance for chaotic light; the parameters $M_{s}$ and $M$ are the usual spatial and temporal degrees of freedom that are well known in the photon-statistics literature. ${ }^{28,29}$ The second set of brackets represents multiplicative noise associated with multiple emissions; $\mathcal{M}$ is the associated degrees-of-freedom parameter. ${ }^{17,18}$ Note that $\langle x\rangle$ multiplies $\beta$ in this term so that it becomes less important as $\langle x\rangle$ decreases. ${ }^{21}$ When each emission is identically a single photon ( $n=1$ Fock state), $\beta$ $=0$, and this contribution vanishes. The third set of square brackets in Eq. (4.6) reflects the fluctuations in the primary excitations. Terms within it disappear when the excitations are Poisson, since then $\rho=0$. The parameters $M_{1}$ and $M_{2}$ represent the degrees-of-freedom parameters associated with the relative widths of the counting time $T$ and combinations of the excitation function $\gamma(t)$ and the emission function $\xi(t)$. Again, it is this contribution that is of essential interest to us here, since we are principally exploring the role of primary excitation statistics in the generation of antibunched and sub-Poisson light. In the following we study the behavior of $M_{1}$ and $M_{2}$ for a number of special cases and in certain special limits.

1. Limiting Case of Instantaneous Emissions $\left(\tau_{p} \ll \tau_{e}\right.$ or $\delta$ $\ll 1)$

When $\tau_{p} \ll \tau_{e}$ [see Eqs. (3.24) and (A9)], $\eta_{1}(\tau)$ and $\eta_{2}(\tau)$ are approximated by Eqs. (3.53) and (3.54), respectively. The expressions for $M_{1}$ and $M_{2}$ in Eqs. (4.9) and (4.10) then simplify considerably and are given by

$$
\begin{aligned}
M_{1}^{-1} & =\frac{2}{T} \int_{0}^{T}(1-\tau / T) \gamma(\tau) \mathrm{d} \tau=M_{e}^{-1}, \\
M_{2} & =M,
\end{aligned}
$$

where $M_{e}$ is the degrees-of-freedom parameter associated with the primary excitation process [see Eq. (A8)]. It then follows from Eq. (4.6) that

$$
\operatorname{Var}(n)=\left[\langle n\rangle+\frac{\langle n\rangle^{2}}{M M_{s}}\right]+\left[\frac{\beta\langle x\rangle}{M}\langle n\rangle\right]+\rho\left[\frac{\langle n\rangle^{2}}{M}+\frac{\langle n\rangle^{2}}{M M_{s}}\right] .
$$

The second and fifth terms, representing wavelike fluctuations, cancel when $\rho=-1$ and/or $M_{s} \rightarrow \infty$. In either case,

$$
\operatorname{Var}(n)=\langle n\rangle+\frac{\beta\langle x\rangle}{\mathcal{M}}\langle n\rangle+\rho \frac{\langle n\rangle^{2}}{M_{e}} .
$$

We now demonstrate that, in this approximation, the photon counts may be viewed as a renewal excitation point process, cascaded with randomly deleted photon emissions (governed by the parameters $\beta$ and $\langle x\rangle$ ).

The Burgess variance formula ${ }^{21,22}$ may be written as

$$
\operatorname{Var}(n)=\langle m\rangle \operatorname{Var}(x)+\langle x\rangle^{2} \operatorname{Var}(m),
$$

where $x$ is the number of photons arising from a single emission that are collected by the detector. The mean of $x$ given by Eq. (4.3), and from Eq. (3.5), its variance is

$$
\operatorname{Var}(x)=\langle x\rangle+(\beta-1)\langle x\rangle^{2},
$$

in the limit of large counting time $T$, where $M=1$. If $m$ is the number of excitations in the time $T$, then, from Eqs. (A6) and (A7),

$$
\begin{aligned}
\langle m\rangle & =\mu T, \\
\operatorname{Var}(m) & =\langle m\rangle+\rho \frac{\langle m\rangle^{2}}{M_{e}},
\end{aligned}
$$

where $M_{e}$ is given by Eq. (A8). Combining Eqs. (4.16)-(4.19) leads to Eq. (4.15), so that, for instantaneous emissions (i.e., in the absence of interference) and for a long counting time $T$, the photon counts do indeed behave as classical particles, as indicated in the previous paragraph.

\section{Example: Instantaneous Emissions with Gamma-2 Excitations}

If the interemission times are described by a gamma distribution of order $\mathcal{N}=2$, then $\rho, \tau_{e}$, and $\gamma(\tau)$ are given in Eq. (A22). Used in conjunction with Eq. (A8), this leads to

$$
M_{e}=2 \theta^{2} /\left(e^{-2 \theta}+2 \theta-1\right)
$$

where

$$
\theta=T / \tau_{e}=2 \mu T=2\langle n\rangle /\langle x\rangle .
$$

If we make use of Eq. (4.15), the ratio between the count variance and the mean (which is known as the index of dispersion or Fano factor $F$ ) is then

$$
F_{n}=\frac{\operatorname{Var}(n)}{\langle n\rangle}=1+\frac{\beta}{M}\langle x\rangle-\frac{\langle n\rangle}{M_{e}},
$$

which, with the help of Eq. (4.20), becomes

$$
\begin{aligned}
F_{n}= & 1+\frac{\beta}{M}\langle x\rangle-\langle x\rangle^{2}[\exp (-4\langle n\rangle /\langle x\rangle) \\
& +4\langle n\rangle /\langle x\rangle-1] / 8\langle n\rangle .
\end{aligned}
$$

In Fig. 12, we plot $F_{n}$ versus $\langle n\rangle$ for several values of $\langle x\rangle$, under the restriction that $\beta=0$. In this case, each emission contains only a single photon, so that the parameter $\langle x\rangle$ represents the collection efficiency of the detector and the 


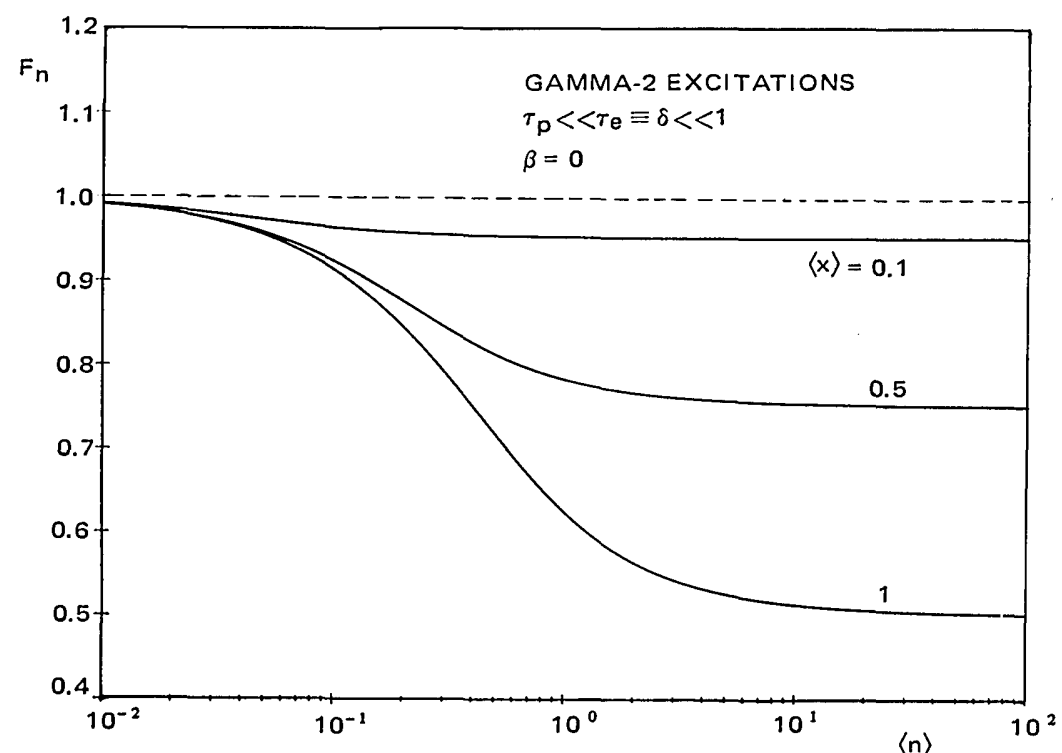

Fig. 12. Fano factor (count variance-to-mean ratio) versus average count $\langle n\rangle$ for gamma-2 excitations with instantaneous emissions $\left(\tau_{p} \ll \tau_{e} \equiv \delta \ll 1\right)$. Single-photon emissions $(\beta=0)$ are assumed. The mean number of detected photons per emission $\langle x\rangle$ is shown as a parameter. The results demonstrate the loss of sub-Poisson behavior introduced by small counting times and random deletion. For $\langle n\rangle \gg 1$ and $\langle x\rangle=$ $1, F_{n} \rightarrow 0.5$, whereas for $\langle x\rangle=0.1, F_{n} \rightarrow 0.95$.

results are independent of $M$. The maximum sub-Poisson behavior is captured for large $T / \tau_{e}$, whereupon $F_{n}=1-$ $\langle x\rangle / 2$. Note that $F_{n}$ is then independent of $\langle n\rangle$, so that the photons behave in a particlelike way. Indeed, we could have formulated our approach in terms of a quantity $\mathcal{M}_{e}$, in analogy with Eq. (4.11) for $M$, rather than in terms of the quantity $M_{e}$ defined by Eq. (A8).

For $\langle x\rangle=1, F_{n}$ varies from a value of 1.0 for small $\langle n\rangle$ to 0.5 for large $\langle n\rangle$, where the particlelike anticlusters are all captured. The light is sub-Poisson. As the collection/detection efficiency $\langle x\rangle$ decreases, $F_{n}$ increases. This of course arises from the additional fluctuations imparted by the independent random deletions associated with the collection and detection processes. The light is Poisson for small $\langle n\rangle$ and sub-Poisson for large $\langle n\rangle$. Note that the condition of validity of Eq. (4.22) is that $\tau_{p} \ll \tau_{e}$, i.e., $\tau_{p} \ll 1 / 2 \mu$ or $\delta=\mu \tau_{p}$ $\ll 0.5$. Therefore, for a given value of $\tau_{p}, \mu$ is upper bounded. Large $\langle n\rangle$ may be attained by using a large counting time $T$. Again, this behavior is identical with that associated with classical particles.

\section{Long-Counting-Time Limit with Many Spatial Modes}

Detected $\left(T \gg \tau_{p}, T \gg \tau_{e}, M_{s} \gg 1\right)$

If the counting time $T$ is much larger than both $\tau_{p}$ and $\tau_{e}$ (or, equivalently, if $\langle n\rangle \gg\langle x\rangle \delta$ and $\langle n\rangle \gg\langle x\rangle)$, Eqs. (4.8)-(4.11) lead to

$$
\begin{aligned}
M & \approx 1, \\
M & \approx T / \tau_{p}^{\prime}, \quad \tau_{p}^{\prime}=2 \int_{0}^{\infty}|c(\tau)|^{2} \mathrm{~d} \tau, \\
M_{1}^{-1} & \approx \frac{2}{T} \int_{0}^{\infty} \eta_{1}(\tau) \mathrm{d} \tau \\
& =\frac{2}{T} \int_{0}^{\infty} \gamma(\tau) \mathrm{d} \tau=M_{e}^{-1}=\tau_{e} / T,
\end{aligned}
$$

$$
\begin{aligned}
M_{2}^{-1} & \approx \frac{2}{T} \int_{0}^{\infty} \eta_{2}(\tau) \mathrm{d} \tau \\
& =\frac{2}{T} \int_{0}^{\infty} \gamma(t)\left|\int_{-\infty}^{\infty} h_{t}\left(t^{\prime}\right) \mathrm{d} t^{\prime}\right|^{2} \mathrm{~d} t,
\end{aligned}
$$

where $\tau_{p}{ }^{\prime}$ is the width of $|c(\tau)|^{2}$. For an exponentially decaying filter, $\tau_{p}{ }^{\prime}=\tau_{p}$. Note that $\int_{0}^{\infty} \eta_{1}(\tau) \mathrm{d} \tau=\int_{0}^{\infty} \gamma(\tau) \mathrm{d} \tau$, independent of the relation between $\tau_{p}$ and $\tau_{e}$. If, furthermore, the area of the detector is sufficiently large that $M_{s} \gg$ 1, the interference terms containing $M_{s}{ }^{-1}$ in Eq. (4.6) may be neglected. This then leads to

$$
\begin{aligned}
\operatorname{Var}(n) & =\langle n\rangle+\beta\langle x\rangle\langle n\rangle+\rho \frac{\langle n\rangle^{2}}{M_{e}} \\
& =\langle m\rangle \operatorname{Var}(x)+\langle x\rangle^{2} \operatorname{Var}(m),
\end{aligned}
$$

where $\operatorname{Var}(x)$ and $\operatorname{Var}(m)$ are given by Eqs. (4.17) and (4.19), respectively. Equation (4.27a) is identical with Eq. (4.15) in the limit $M=1$. The Burgess variance formula is again satisfied under these conditions, and the photons behave like classical particles.

If, in addition, $\beta=0$ and $\rho=-1$,

$$
F_{n}=1-\mu \tau_{e}\langle x\rangle \text {, }
$$

where of course $\mu \tau_{e} \leq 1$ and $\langle x\rangle \leq 1$. This is a simple and useful result, which bespeaks essentially optimal sub-Poisson behavior. We may associate an overall effective efficiency $\eta_{\text {eff }}$ with the quantity $\mu \tau_{e}\langle x\rangle$, and an effective number of trials $N$ with $T / \tau_{e}$, whereupon Eq. (4.2) indicates that $\langle n\rangle=\eta N$, Eq. (4.27a) shows that $\operatorname{Var}(n)=\langle n\rangle\left(1-\eta_{\text {eff }}\right)$, and Eq. (4.27b) provides that $F_{n}=\left(1-\eta_{\mathrm{eff}}\right)$. These statistics are presented in the usual form for a binomial distribution. For gamma- $\mathcal{N}$ excitations, Eq. (4.27b) becomes $F_{n}=1-\langle x\rangle+(\langle x\rangle / \mathcal{N})$. When $\langle x\rangle=1$, the result is $F_{n}=1 / \mathcal{N}$, whereas when $\mathcal{N} \rightarrow \infty$, the outcome is $F_{n}=1-\langle x\rangle$. This latter result is the most usual form for the binomial and is in accord with Eq. (10) of 
Ref. 5. For pulse-train excitations, Eq. (4.27a) shows that sub-Poisson behavior can be achieved only for $\beta<1$.

\section{Long-Counting-Time Limit with Exponentially}

Decaying Emissions $\left(T \gg \tau_{p}, \tau_{p}^{\prime} ; T \gg \tau_{e}\right.$ )

When $T \gg \tau_{p}, \tau_{p}{ }^{\prime}$ and $T \gg \tau_{e}$, the degrees-of-freedom parameters may be approximated by expressions (4.23)-(4.25). We now fold in the additional restriction that $\xi(t)$ be exponential, as in Eq. (3.25), but relax the condition that $M_{s}=\infty$. Substituting Eqs. (3.25)-(3.29) in expression (4.26) yields

$$
M_{2}^{-1}=\frac{1}{T} \int_{0}^{\infty} \gamma(t) \exp \left(-2 t / \tau_{p}\right) \mathrm{d} t=1 / 2 \frac{\tau_{p}}{T} \eta_{1}(0),
$$

where Eq. (3.31) has been used. $\eta_{1}(0)$ is given by Eq. (3.32), and by Eqs. (3.33), (3.36), and (3.39) for the special primary excitation processes considered earlier. The count variance in Eq. (4.6) is then given by

$$
\begin{aligned}
\operatorname{Var}(n)= & \langle n\rangle+\frac{\langle n\rangle^{2}}{M_{s} T / \tau_{p}^{\prime}}+\beta\langle x\rangle\langle n\rangle \\
& +\rho\left[\frac{\langle n\rangle^{2}}{T / \tau_{e}}+\frac{\langle n\rangle^{2}}{2 M_{s} T / \tau_{p}} \eta_{1}(0)\right],
\end{aligned}
$$

which corresponds to a Fano factor

$$
F_{n}=\left[1+\left(\beta+\rho \mu \tau_{e}\right)\langle x\rangle\right]+\frac{\langle x\rangle}{M_{s}}\left[\delta^{\prime}+\frac{1}{2} \rho \eta_{1}(0) \delta\right],
$$

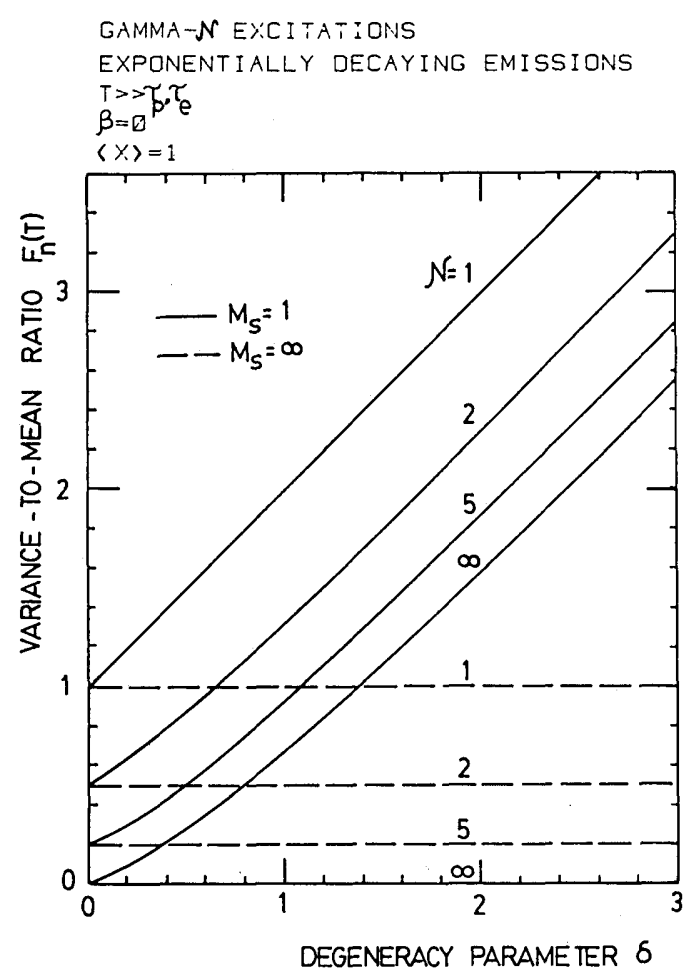

(a) where $\delta^{\prime}=\mu \tau_{p}^{\prime}$

As an example, we consider gamma- $\mathcal{N}$ excitations. If we use Eq. (A22) and $\rho=-1$ together with Eq. (3.33), Eq. (4.30) reduces to

$$
F_{n}=1+\left[\beta-\left(1-\frac{1}{\mathcal{N}}\right)\right]\langle x\rangle+\frac{\langle x\rangle}{M_{s}}\left[\frac{1}{\left(1+\frac{2}{\mathcal{N} \delta}\right)^{\mathcal{N}}-1}+\frac{\delta}{2}\right] .
$$

Assuming further that $\beta=0$ and $\langle x\rangle=1$ leads to the result that

$$
F_{n}=\frac{1}{\mathcal{N}}+\frac{1}{M_{s}}\left(\frac{1}{(1+2 / \mathcal{N} \delta)^{\mathcal{N}}-1}+\frac{\delta}{2}\right) .
$$

Furthermore, if a large number of spatial modes are detected, $M_{s} \rightarrow \infty$, and we recover the simple result that

$$
F_{n}=1 / \mathcal{N}
$$

in accord with the expression for the gamma- $\mathcal{N}$ primary renewal excitation process [see Eq. (A24)]. This is again a situation in which the classical behavior of the primary excitations is transferred directly to the photons.

The dependence of the Fano factor $F_{n}$ on the degeneracy parameter $\delta$ [Eq. (4.32)] is shown in Fig. 13(a) with $\mathcal{N}$ as a parameter, when $M_{s}=1$ (solid curves) and $M_{s}=\infty$ (dashed

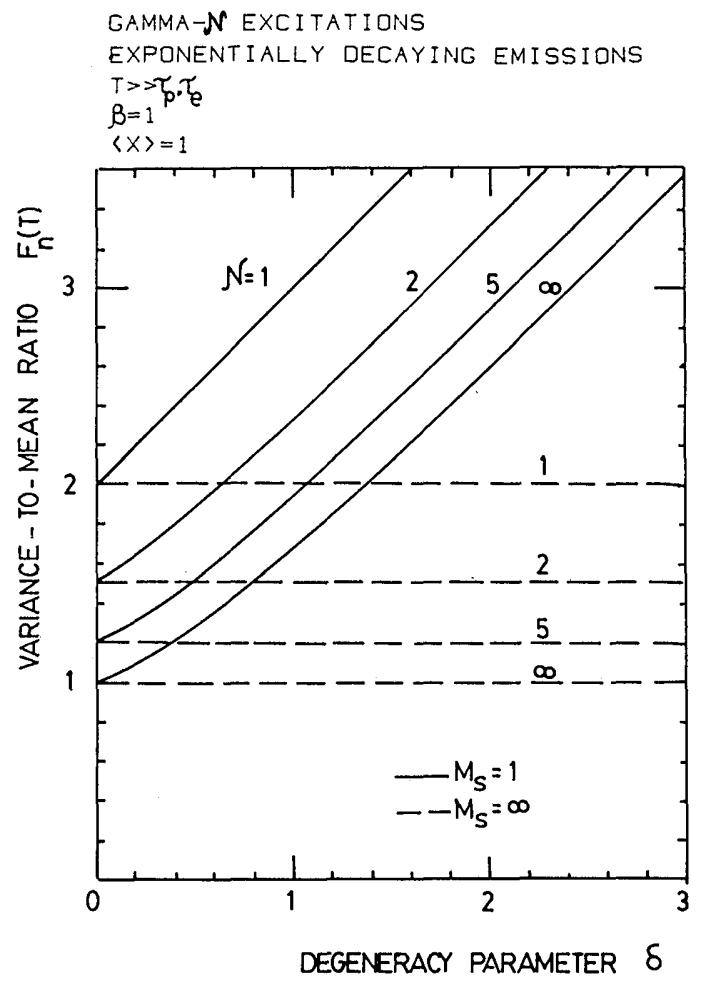

(b)

Fig. 13. Fano factor $F_{n}$ versus degeneracy parameter $\delta$ for gamma- $\mathcal{N}$ excitations in the long-counting-time limit $\left(T \gg \tau_{p}, \tau_{e}\right)$. The emissions are assumed to be exponentially decaying. The mean number of detected photons per emission $\langle x\rangle=1$. Solid curves represent the detection of a single spatial mode $\left(M_{s}=1\right)$, whereas dashed curves represent the detection of a large number of spatial modes $\left(M_{s}=\infty\right)$. (a) Single-photon emissions $(\beta=0)$. Note that the photon-counting distribution will be sub-Poisson $\left(F_{n}<1\right)$ for all $\delta$, provided that $\mathcal{N}>1$ and $M_{s}=\infty$. (b) Poisson photon emissions $(\beta=1)$ with an average of one photon per emission $(\langle x\rangle=1)$. Note that the photon-counting distribution will be super-Poisson $\left(F_{n}>1\right)$ for $\mathcal{N}<\infty$. 
curves). In all cases $\beta=0$ and $\langle x\rangle=1$. For $M_{s}=1$, the light is seen to be sub-Poisson only when $\delta$ is small. For the $\mathcal{N}=$ $\infty$ curve, it is clear that $F_{n}<1$ for $\delta \lesssim 1.4$, whereas from Fig. 5 we see that $g^{(2)}(0)<1$ for $\delta<1.6$. Thus, when $1.4 \lesssim \delta \lesssim 1.6$, the light is antibunched but super-Poisson. This is not prohibited, as we pointed out previously, ${ }^{5}$ since $g^{(2)}(\tau)$ is nonmonotonic, as is seen from Fig. 10. For $\mathcal{N}=2, F_{n}<1$ for $\delta \lesssim$ 0.6 , whereas $g^{(2)}(0)<1$ for $\delta \lesssim 0.5$. When $\delta$ is large, wave fluctuation noise drives the light toward super-Poisson behavior, in spite of the primary sub-Poisson excitations (for $\mathcal{N}$ $>1$ ). For $\mathcal{N}=1, F_{n}=1+\delta$, as expected. It is important to note that the wave fluctuations are suppressed as $M_{s}$ increases; indeed, as $M_{s} \rightarrow \infty$, the light remains sub-Poisson for arbitrarily large values of the degeneracy parameter. This means that an intense source of sub-Poisson light $\left(\mu \tau_{p} \gg 1\right)$ can be realized if many spatial modes are detected.

The result for Poisson emissions, with an average of one photon per emission $(\beta=1,\langle x\rangle=1)$ is shown in Fig. 13(b) for comparison. As is evident from Eq. (4.31), sub-Poisson behavior is no longer possible under any conditions $\left(F_{n} \geq 1\right)$. The Poisson nature of the individual emissions contributes an irreducible randomness, as displayed schematically in Fig. 1. For $\mathcal{N}=1$ (Poisson excitations) $F_{n}=2+\delta$, in accord with the proper result for this special case of the Neyman Type-A distribution. ${ }^{18}$

\section{Short-Counting-Time Limit with One Spatial Mode} Detected $\left(T \ll \tau_{p}, T \ll \tau_{e}, M_{s} \approx 1\right)$

In the opposite limit of short counting time and small detector area, Eqs. (4.8)-(4.11) yield

$$
\begin{aligned}
M & \approx 1, \quad M_{1}^{-1} \approx \eta_{1}(0), \\
M_{2}^{-1} & \approx \eta_{2}(0) \approx \eta_{1}(0), \\
M & \approx \tau_{p} / T,
\end{aligned}
$$

and, by assumption,

$$
M_{s} \approx 1
$$

This leads to a count variance from Eq. (4.6) given by

$$
\begin{aligned}
\operatorname{Var}(n) & =\langle n\rangle+\langle n\rangle^{2}+\beta\langle x\rangle \frac{T}{\tau_{p}}\langle n\rangle+2 \rho \eta_{1}(0)\langle n\rangle^{2} \\
& =\langle n\rangle+\langle n\rangle^{2}\left[1+\frac{\beta}{\delta}+2 \rho \eta_{1}(0)\right] .
\end{aligned}
$$

By using Eq. (3.30), this can be written as

$$
\operatorname{Var}(n)=\langle n\rangle+\langle n\rangle^{2}\left[g^{(2)}(0)-1\right] ;
$$

Eq. (4.37) is of course consistent with Eq. (4.4) for short $T$ and small $A$. In this case, the behavior of $\operatorname{Var}(n)$ is determined directly by the behavior of $g^{(2)}(0)$, so that bunching/antibunching corresponds to super-/sub-Poisson behavior, as illustrated previously for time behavior alone. ${ }^{5}$

\section{Pulse-Train Excitations with Exponentially Decaying Emissions}

When the excitation is a train of periodic pulses with random phase, Eq. (A34) can be used in Eqs. (3.18), (3.19), (4.9), and (4.10) to determine $M_{1}$ and $M_{2}$. The result is that

$$
\begin{aligned}
& M_{1}^{-1}=1-\frac{2 T_{0}}{T} \int_{0}^{T}(1-\tau / T) \sum_{l \neq 0} \bar{h}_{0}\left(\tau-l T_{0}\right) \mathrm{d} \tau, \\
& M_{2}^{-1}=M^{-1}-\frac{2 T_{0}}{T} \int_{0}^{T}(1-\tau / T) \sum_{l \neq 0} \bar{h}_{\tau}\left(l T_{0}\right) \mathrm{d} \tau .
\end{aligned}
$$

When $\xi(t)$ is exponential, Eq. (3.27) yields

$$
\begin{aligned}
M_{1}^{-1}= & 1+\frac{2}{\delta} M^{-1}-\frac{2 / \delta}{1-e^{-2 / \delta}} \frac{1}{T} \int_{0}^{T}(1-\tau / T) \\
& \times(\exp [-2 \theta(\tau) / \delta]+\exp \{-2[1-\theta(\tau)] / \delta\}) \mathrm{d} \tau, \\
M_{2}^{-1}= & {\left[1-\frac{2 / \delta}{e^{2 / \delta}-1}\right] M^{-1}, }
\end{aligned}
$$

where $\theta(t)$ is the periodic triangular function defined by Eq. (3.49).

In the limit $\delta \gg 1$, Eq. (4.41) provides that $M_{1}^{-1} \approx 0, M_{2}{ }^{-1}$ $\approx 0$, so that when $\beta=0$, Eq. (4.6) becomes

$$
\operatorname{Var}(n)=\langle n\rangle+\frac{1}{M M_{s}}\langle n\rangle^{2},
$$

as for ordinary chaotic light. In the limit $\delta \ll 1$, on the other hand,

$$
\begin{aligned}
M_{1}^{-1} & \approx \frac{1}{\mu T}-\left(\frac{1}{\mu T}\right)^{2} \theta_{0}\left(1-\theta_{0}\right), \\
M_{2} & \approx M,
\end{aligned}
$$

where $\theta_{0}=\theta(T)$ is given by Eq. (A36). Taking $\beta=0$, we have

$$
\begin{aligned}
\operatorname{Var}(n) & =\langle n\rangle-\left[\langle x\rangle\langle n\rangle-\langle x\rangle^{2} \theta_{0}\left(1-\theta_{0}\right)\right] \\
& =\mu T\langle x\rangle(1-\langle x\rangle)+\langle x\rangle^{2} \theta_{0}\left(1-\theta_{0}\right) .
\end{aligned}
$$

For $\langle x\rangle=1$, this is simply

$$
\operatorname{Var}(n)=\theta_{0}\left(1-\theta_{0}\right)
$$

reproducing Eq. (A35). In this limit $(\delta \ll 1, \beta=0,\langle x\rangle=1)$, the primary excitation statistics are transferred directly to the photons. Choosing a value $\langle x\rangle\langle 1$ introduces random deletion of the photons through spatial and efficiency loss. Thus Eq. (4.44) represents the count variance for a randomly deleted periodic pulse train of random phase.

\section{B. Counting Probability Distribution}

The probability distribution of the number of photons $n$ crossing a given area $A$ in the time interval $[0, T]$ is related to the field operators by 29

$$
p_{n}(n)=\left\langle: \hat{W}^{n} e^{-W} / n !:\right\rangle,
$$

where

$$
\hat{W}=\int_{A} \int_{0}^{T} \hat{E}^{-}(\mathbf{r}, t) \hat{E}^{+}(\mathbf{r}, t) \mathrm{d} \mathbf{r} \mathrm{d} t .
$$

The symbols : : represent (time and) normal ordering of the operators. In our case, $E^{+}(\mathbf{r}, t)$ is given by Eqs. (2.1), (2.2), and (2.3). The operation of averaging in Eq. (4.46) represents a quantum-mechanical expectation as well as a classical average over the random excitation times $\left\{t_{k}\right\}$ and locations $\left\{\mathbf{r}_{k}^{\prime}\right\}$. It is apparent that the problem of evaluating Eq. (4.46) is formidable. 
Under certain special conditions, however, $p(n)$ may be determined from simple arguments. If the counting time $T$ is long in comparison with the emission lifetime $\tau_{p},\left(T \gg \tau_{p}\right)$, and if the total mean number of emissions in the interval $T$ is large $\left(T \gg \mu^{-1}\right)$, almost all emissions occurring in the time interval $[0, T]$ produce photons in the same interval, provided also that the detector area is sufficiently large. These are essentially the conditions of Section 4. A.3. The number of collected photons $n$ is then determined from the number of emissions $m$ by first assigning to each emission a random number of photons $n_{0}$. The outcome is then randomly deleted by a Bernoulli process of success probability $\eta=$ $\int_{A} D^{(1)}(\mathbf{r}, \mathbf{r}) \mathrm{d} \mathbf{r}$, which is the collection/registration efficiency in accordance with Eq. (4.3). The statistics of the number of emissions $m$ is, of course, obtained from renewal theory (see Appendix A). Excitations may not always result in emissions, but a randomly deleted renewal process remains a renewal process, ${ }^{30}$ so we have lost no generality by assuming that all excitations result in emissions.

If $G_{m}(z), G_{n 0}(z)$, and $G_{n}(z)$ are the probability-generating functions of $m, n_{0}$, and $n$, respectively $\left[G_{x}(z)=\left\langle z^{x}\right\rangle\right]$, then ${ }^{21,22}$

$$
G_{n}(z)=G_{m}\left[G_{n 0}(1-\eta+\eta z)\right] .
$$

The probability distribution $p_{n}(n)$ may be obtained from $G_{n}(z)$ by using the standard rule 28 :

$$
p_{n}(n)=\left.\frac{1}{n !} \frac{\partial^{n}}{\partial z^{n}} G_{n}(z)\right|_{z=0 .}
$$

In the following we consider two simple cases.

1. $p_{n}(n)$ for Long Counting Time and Single-Photon Emissions $(T \rightarrow \infty, \beta=0)$

If $n 0=1$, i.e., emissions are in the $n=1$ Fock state, $\beta=0$ and $G_{n 0}(z)=z$. In that case Eq. (4.48) gives

$$
G_{n}(z)=G_{m}(1-\eta+\eta z) .
$$

The photon-counting distribution $p_{n}(n)$ is then a randomly deleted version of the distribution of the number of primary excitations, $p_{m}(m)$, so that Eq. (4.50) is equivalent to ${ }^{22}$

$$
p_{n}(n)=\sum_{k=n}^{\infty}\left(\begin{array}{l}
k \\
n
\end{array}\right) \eta^{n}(1-\eta)^{k-n} p_{m}(k) .
$$

In the simplest situation in which the primary excitations arise from a periodic pulse train [i.e., $p_{m}(k)=\delta_{k, m 0}$ or $k=m_{0}$ deterministically],

$$
p_{n}(n)=\left(\begin{array}{c}
m_{0} \\
n
\end{array}\right) \eta^{n}(1-\eta)^{m_{0}-n},
$$

which is the binomial distribution. 5,31

If the primary excitations are instead described by the more general gamma- $\mathcal{N}$ renewal process, $p(m)$ is given by Eq. (A26). This distribution is shown graphically in Fig. 14(a) for a fixed mean count $\langle m\rangle=10$, with $\mathcal{N}$ as a parameter. Curves are shown for $\mathcal{N}=1$ (solid curve, $F_{n}=1.0$ ); $\mathcal{N}=2$ (dasheddotted curve, $F_{m}=0.52$ ), and $\mathcal{N}=4$ (dashed curve, $F_{m}=$ $0.27)$. The curve for $\mathcal{N}=1$ is the Poisson. The gamma- $\mathcal{N}$ counting distribution is always sub-Poisson for $\mathcal{N}>1$, narrowing as $\mathcal{N}$ increases in accordance with the approximate variance-to-mean ratio $F_{m} \approx 1 / \mathcal{N}$ given in Eq. (A24). In the limit $\mathcal{N} \rightarrow \infty, p_{m}(m)$ becomes a delta-function counting distribution.

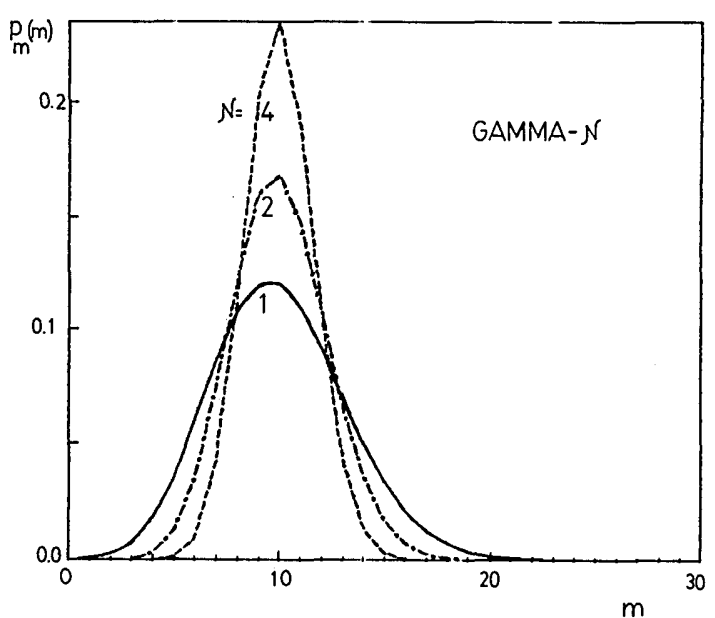

(a)

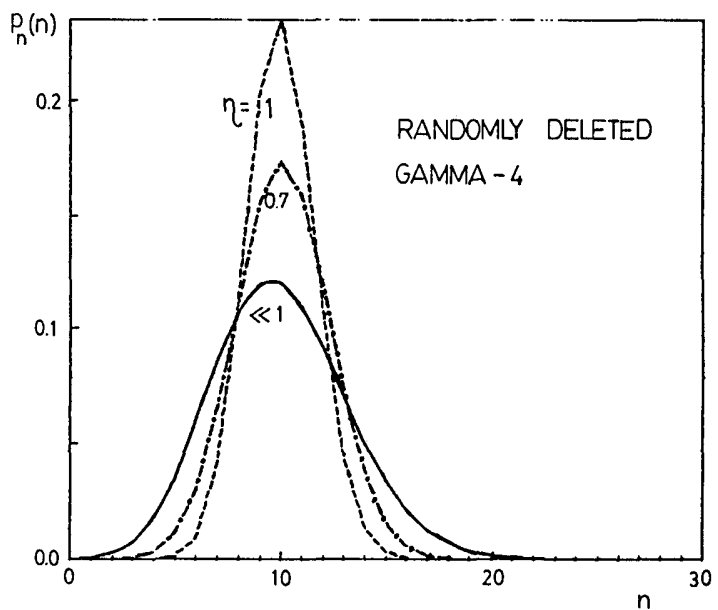

(b)

Fig. 14. (a) Excitation-counting probability distribution $p_{m}(m)$ versus count number $m$ for the gamma- $\mathcal{N}$ counting process in the limit of long counting times. The mean excitation count $\langle m\rangle=10$, and $\mathcal{N}$ is a parameter. $\mathcal{N}=1$ (solid curve; Poisson distribution); $\mathcal{N}$ $=2$ (dashed-dotted curve); $\mathcal{N}=4$ (dashed curve). All these distributions are sub-Poisson for $\mathcal{N}>1$. As $\mathcal{N} \rightarrow \infty, p_{m}(m)$ approaches a $\delta$ function. (b) Photon-counting probability distribution $p_{n}(n)$ versus count number $n$ for the randomly deleted gamma- 4 counting process in the limit of long counting times. This result is appropriate for single-photon emissions $(\beta=0)$. All these distributions are always sub-Poisson. The mean photon count $\langle n\rangle=\eta\langle m\rangle=10$, and $\eta$ is the deletion parameter. $\eta \ll 1$ (solid curve; Poisson distribution); $\eta=$ 0.7 (dashed-dotted curve); $\eta=1$ (dashed curve; gamma-4 distribution).

The deleted distribution $p_{n}(n)$ can be obtained in numerical form from Eqs. (4.51) and (A26). The outcome will remain a renewal-process counting distribution. ${ }^{30}$ The result is illustrated in Fig. 14(b) for $\mathcal{N}=4$ and $\langle n\rangle=\eta\langle m\rangle=10$ The deletion parameter $\eta$ takes on three values: $\eta \ll 1$ (solid curve, $F_{n}=1.0$ ); $\eta=0.7$ (dashed-dotted curve, $F_{n}=0.50$ ); and $\eta=1$ (dashed curve, $F_{n}=0.27$ ). In the limit $\eta \rightarrow 0$, all such distributions approach the Poisson, so the solid curve is identical with the solid curve in Fig. 14(a). In the limit $\eta=$ 1 , all emitted photons are collected and counted, so that the distributions of $n$ and $m$ become identical. Thus the dashed curve in Fig. 14(b) is the same as the dashed curve in Fig. 14(a), viz., the gamma-4 counting distribution. For intermediate values of $\eta, F_{n}$ lies between the two extremes, i.e., $1 / \mathcal{N} \lesssim F_{n}$ 
$\lesssim 1$, as is evident from the dashed-dotted curve. These distributions will always be sub-Poisson. ${ }^{21}$ Increasing $\mathcal{N}$, with $\eta$ and $\langle n\rangle$ held fixed, narrows the distribution.

\section{2. $p_{n}(n)$ for Long Counting Time and Quasi-Coherent}

Emissions $(T \rightarrow \infty, \beta=1)$

If each emission is in a coherent state, $n 0$ has a Poisson distribution and $\beta=1$. In this case

$$
G_{n 0}(z)=\exp [(z-1) \bar{n} 0],
$$

so that the result for the probability-generating function is

$$
G_{n}(z)=G_{m}\{\exp [(z-1) \eta \bar{n} 0]\} .
$$

Using the rule for conditional probability distributions, ${ }^{18}$ we obtain

$$
p_{n}(n)=\sum_{m=0}^{\infty} \frac{[\eta \bar{n} 0 m]^{n} \exp [-\eta \bar{n} 0 m]}{n !} p_{m}(m) .
$$

It is clear that the photon-counting distribution $p_{n}(n)$ can be computed for an arbitrary excitation-counting distribution $p_{m}(m)$. If the excitations $m$ are themselves Poisson, the photon-counting distribution $p_{n}(n)$ is the Neyman Type-A distribution that we have discussed extensively elsewhere. ${ }^{18}$ If the excitations follow the binomal distribution, the photon-counting distribution becomes the binomial/Poisson distribution.

If the excitations are characterized by a gamma- $\mathcal{N}$ renewal process, then the distribution $p_{m}(m)$ represented in Eq. (A26) can be used in Eq. (4.55) to compute the photon-counting distributions. The character of the family of gamma$\mathcal{N} /$ Poisson distributions is illustrated graphically in Fig. 15 for various values of $\mathcal{N}$ and $\langle x\rangle=\eta \bar{n} 0$. In Fig. 15(a), we present the gamma- $\mathcal{N} /$ Poisson distribution with $\langle x\rangle=\eta \bar{n} 0$ $=5.0$ and with $\langle m\rangle=2$, to provide a final mean count fixed at $\langle n\rangle=10 . \mathcal{N}$ takes on the values 1 (solid curve), 4 (dashed-dotted curve), and 10 (dashed curve). The Fano factor $F_{n}$ for the three curves is 6.0,2.7, and 1.8, respectively. The result for $\mathcal{N}=1$ corresponds to the Neyman Type-A distribution, ${ }^{18}$ for which $F_{n}=1+\langle x\rangle$. As $\mathcal{N} \rightarrow \infty$, the primary process increasingly resembles a random-phase pulse train, so that $p_{n}(n)$ approaches the Poisson limit. In Fig. $15(b), \mathcal{N}$ is fixed at 2 , and $\langle x\rangle$ and $\langle m\rangle$ are varied, subject to the constraint that the product $\langle n\rangle=\langle x\rangle\langle m\rangle=10$. The solid curve corresponds to $\langle x\rangle=10,\langle m\rangle=10, F_{m}=1.5$; the dashed-dotted curve to $\langle x\rangle=5,\langle m\rangle=2, F_{n}=4.0$; and the dashed curve to $\langle x\rangle=1,\langle m\rangle=1, F_{n}=7.7$. The Fano factor increases as $\langle x\rangle$ increases but always remains below the corresponding value for the Neyman Type-A distribution. This is because the gamma-2 primary distribution is narrower than the Poisson of identical mean.

All these distributions are always super-Poisson, as is apparent from Fig. 1 and Eq. (4.31). An expression for the gamma- $\mathcal{N} /$ Poisson Fano factor $F_{n}$ can be obtained by using the asymptotic approximation for $F_{m}$ in Eq. (A24), together with the Burgess variance theorem. ${ }^{21,22}$ The result is that

$$
F_{n} \approx 1+\eta \bar{n}_{0} / \mathcal{N} \text {. }
$$

$F_{n}$ approaches unity for $\eta \bar{n} 0 \rightarrow 0$ and/or $\mathcal{N} \rightarrow \infty$, corresponding to heavy random deletion and/or pulse-train excitations, respectively. Expression (4.56) behaves similarly to the Fano factor for the shot-noise-driven doubly stochastic

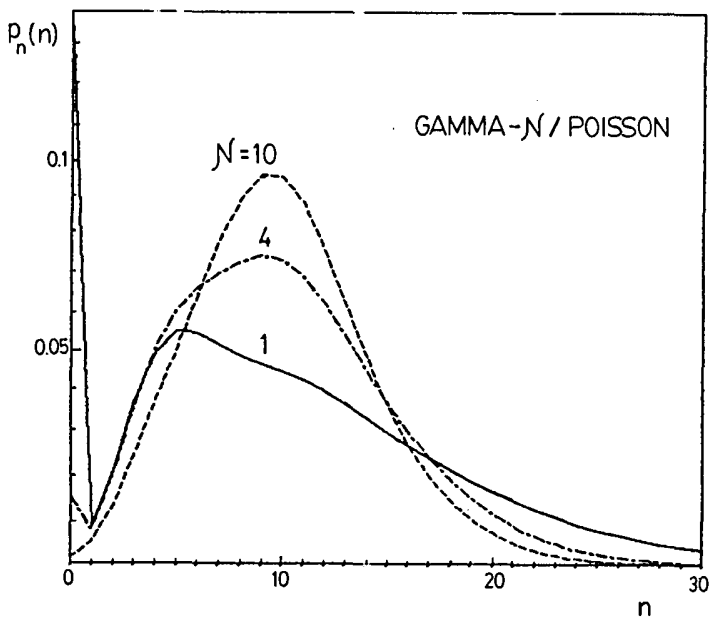

(a)

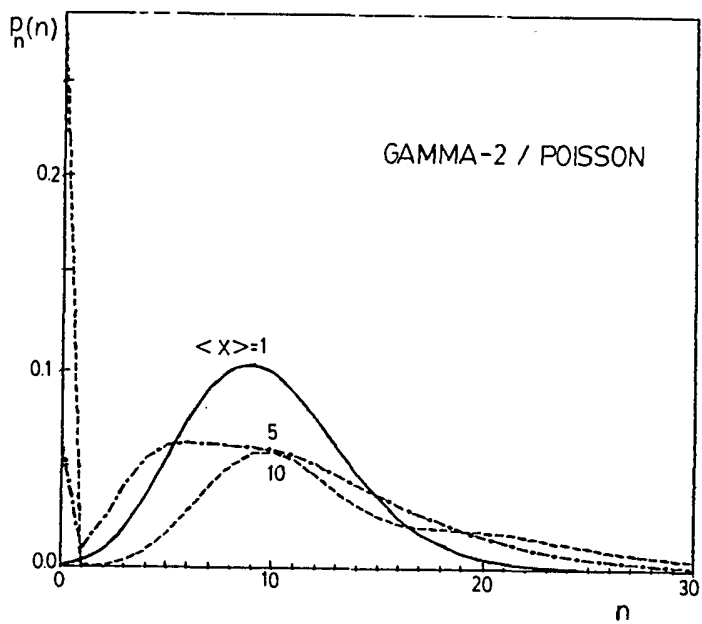

(b)

Fig. 15. Photon-counting probability distribution $p_{n}(n)$ versus count number $n$ for the gamma- $\mathcal{N} /$ Poisson counting process in the limit of long counting times. This result is appropriate for quasi-coherent individual emissions $(\beta=1)$. All these distributions are always super-Poisson. (a) $\langle x\rangle=\eta \bar{n}_{0}=5,\langle m\rangle=2,\langle n\rangle=10$, with $\mathcal{N}$ as a parameter. $\mathcal{N}=1$ (solid curve; Neyman Type-A distribution); $\mathcal{N}=$ 4 (dashed-dotted curve); $\mathcal{N}=10$ (dashed curve). As $\mathcal{N} \rightarrow \infty, p_{n}(n)$ approaches the Poisson. (b) $\mathcal{N}=2,\langle n\rangle=\langle x\rangle\langle m\rangle=10$, with $\langle x\rangle$ and $\langle m\rangle$ as parameters. $\langle x\rangle=1,\langle m\rangle=10$ (solid curve); $\langle x\rangle=5$, $\langle m\rangle=2$ (dashed-dotted curve); $\langle x\rangle=10,\langle m\rangle=1$ (dashed curve).

Poisson process (SNDP), ${ }^{21}$ where $\mathcal{N}$ plays the role of the degrees-of-freedom parameter $\mathcal{M}$; however, here large $\mathcal{N}$ is associated with a narrow counting distribution for the primary process, whereas large $\mathcal{M}$ in the SNDP is associated with the cutting apart of particlelike clusters in the final point process.

\section{CONCLUSION}

There are three distinct measures that have been used in modern optics for characterizing the statistical nature of a source of light. These are defined by the terms (1) bunched/antibunched, (2) super-Poisson/sub-Poisson, and (3) nonsqueezed/squeezed. Each corresponds to a different experimental paradigm, viz., (1) coincidence detection, (2) photo-counting detection, and (3) heterodyne detection, respectively.' These manifestations of nonclassical behavior can exist in various combinations, depending on the particular 
nature of the radiation. ${ }^{5}$ Classical light is bunched, superPoisson, and nonsqueezed. If one or more of the complementary conditions are obeyed, the light is defined as nonclassical. Ideal laser light lies just at the bottom edge of the classical boundary: It is neither bunched nor antibunched $\left[g^{(2)}(\tau)=1\right.$ for all $\left.\tau\right]$, it is precisely Poisson $\left[F_{n}(T)=1\right.$ for all $T]$, and it is not squeezed.

We have studied the antibunching and sub-Poisson properties of nonclassical light generated by the two-step process of excitation followed by emission. Antibunching is defined in terms of the normalized second-order correlation function of the light and represents the two-photon coincidence probability. It was one of the earliest parameters to be investigated in connection with the coherence properties of classical light, ${ }^{2}$ and it continues to find use in applications such as photon-correlation spectroscopy and the determination of the diameters of stars (for which the light is assumed to be thermal). Our principal focus in the following is on the behavior and applications of sub-Poisson light. The individual emissions in our examples are all nonsqueezed, leading us to expect that the stationary light generated by the superposition of these emissions will also be nonsqueezed.

Sub-Poisson (or quiet) light appears to have several potential areas of usefulness. Two of these are (1) as an improved source for investigating physical and biological processes, such as the behavior of the mammalian and nonmammalian visual system at threshold, ${ }^{8}$ and (2) as an improved source for use in signal processing and direct-detection fiber-optic communication systems. Its allure stems from the low value of the count variance-to-mean ratio $F_{n}(T)$. This measure succinctly describes the noisiness of a light source (in terms of noise-to-signal ratio) relative to that of the ideal amplitude-stabilized laser, for which it is unity for all $T$. It follows that the probability of error, which characterizes the performance of a communication system, will be reduced by the use of sub-Poisson light. Potential applications, such as those cited above, provide added motivation for studying the mechanisms involved in generating such radiation.

We have discussed the conditions required to produce strong sub-Poisson light by means of a two-step process. These are (1) the use of sub-Poisson (classical) excitation statistics, (2) the generation of independent one-photon (quantum-mechanical) emissions, and (3) the avoidance (or averaging) of interference effects. Under these circumstances, the classical primary excitation statistics will be directly transferred to the photons. This is true both for the second-order correlation function and for the photon counts. The process may be viewed as the inverse of photodetection, in which the statistical character of photons is transferred to photoelectron counts.

A way of achieving these conditions is provided by the Franck-Hertz experiment, as we have recently reported. ${ }^{5}$ If space charge is used to regularize the spacing of the electrons in their travels through the passive atomic medium, the excitation statistics are rendered sub-Poisson. Emissions then occur in the form of one-photon states as the excited atoms spontaneously decay from their first excited state to the ground state $\left(6^{3} P_{1}-6^{3} S_{0}\right.$ in $\left.\mathrm{Hg}\right)$. Finally, the light is collected from a large solid angle. This provides the spatial integration that averages the interference, thereby allowing the degeneracy parameter to be large and the source to be intense. An elementary treatment of this process has already been pro- vided $^{5}$; the results of the more complete model considered here reduce to those obtained in Ref. 5 if it is assumed that the emissions occur instantaneously $\left(\tau_{p} \ll \tau_{e}\right)$.

If any of the three conditions cited above is violated, the nonclassical nature of the light is compromised. Indeed, super-Poisson (or even Poisson) excitations, and/or classical emissions (coherent or thermal), always result in bunched and super-Poisson light. If the excitations are ideally sub-Poisson and single-photon emissions ensue, but interference effects are not averaged, sub-Poisson behavior is possible only for weak sources (low values of the degeneracy parameter). Of course, if the number of steps in the process is increased beyond two (as it is in an optical amplifier, for example), the initial sub-Poisson behavior will be diminished. ${ }^{32,33}$

There are many methods for generating light, and it is interesting to see how our analysis may relate to them. The laser, which is a highly nonlinear device, links the photon emissions together by means of stimulated emission. These emissions are therefore not independent, so that our model will not apply. It would be interesting to study the effect of excitation statistics on the coherence properties of light generated in the presence of stimulated emission and saturation.

The generation of antibunched and sub-Poisson light by means of nonlinear optical processes also does not fit the model of our analysis. As an example, we consider singleatom resonance fluorescence because it is the only effect that has thus far successfully been used to generate nonclassical light. ${ }^{11,15}$ In this case, the (laser-photon) excitations are Poisson; it is the nonindependence of the one-photon emissions that allows the effect to occur. This is because the atom returns to its ground state when a photon is emitted and cannot immediately reradiate. For resonance fluorescence radiation to behave in this manner, however, there must be only a single atom in the field of view at any one time. Fluctuations in the number of active radiators work against the effect. ${ }^{11,15-17}$ So too does the Poisson convergence theorem, ${ }^{30,34-37}$ which holds that the superposition of a large number of independent, stationary, orderly processes asymptotically approaches the Poisson process under suitable conditions of sparseness.

There are a number of extensions of our model that could prove useful. For simplicity, we have chosen renewal-process excitation times and (separable) uniformly distributed radiator positions. Other processes that take on sub-Poisson behavior could be used to model the excitations. For the same reasons (viz., simplicity of analysis), we have assumed that the individual emissions are statistically independent, identical, and single mode (though not necessarily monochromatic). The state of the emissions was chosen to be coherent, chaotic, or $n$-state. These assumptions may be relaxed, leading to a study of light whose emissions may be correlated, multimode, and/or in states other than those cited above.

There are various limiting conditions on the calculations that we have carried out. Correlation functions higher than second order could be computed, providing expressions for higher-order multiple-photon-coincidence counting rates. These would be interesting for some of the more unusual sources of light that we have examined. Finally, our mathematical investigation of the photon-counting distributions has been sketchy. Only some rough characterizations have been provided, and these are based on heuristic reasoning. An 
atiempt should be made to derive proper distributions analytically for arbitrary values of the counting time $T$.

\section{APPENDIX A: SOME SALIENT PROPERTIES OF RENEWAL PROCESSES}

A renewal process is a point process with independent interevent times. If the process is stationary, the interevent times are identically distributed and the process is characterized completely by the probability density function $f(\tilde{\tau})$ of the interevent time $\tilde{\tau}$. In this appendix we review some of the properties of stationary renewal processes. ${ }^{19}$

\section{A. Density and Covariance Function}

The density and the covariance function of a point process are defined by

$$
\begin{aligned}
\mu & =\lim _{\Delta t \rightarrow 0+}\left\langle N_{\Delta t}(t)\right\rangle / \Delta t, \\
\sigma(\tau) & =\lim _{\Delta t \rightarrow 0+}\left[\left\langle N_{\Delta t}(t) N_{\Delta t}(t+\tau)\right\rangle-\left\langle N_{\Delta t}(t)\right\rangle^{2}\right] /(\Delta t)^{2},
\end{aligned}
$$

respectively, where $N_{T}(t)$ is the number of events in the interval $(t, t+T)$.

For a renewal process,

$$
\begin{aligned}
\mu & =1 /\langle\tilde{\tau}\rangle, \\
\sigma(\tau) & =\mu \delta(\tau)+\mu^{2} \rho \gamma(\tau),
\end{aligned}
$$

where

$$
\rho \gamma(\tau)=\frac{1}{\mu} q(\tau)-1, \quad \rho=\frac{1}{\mu} q(0)-1,
$$

and $q(\tau)$ is a symmetric function $[q(-\tau)=q(\tau)]$ satisfying

$$
q(\tau)=\bar{q}(\tau), \quad \tau \geq 0 .
$$

The function $q(\tau)$ is the inverse Laplace transform of

$$
\bar{Q}(s)=Q(s) /[1-Q(s)],
$$

where $Q(s)$ is the Laplace transform of $f(\tilde{\tau})$.

\section{B. Counting Statistics}

The mean and the variance of the number of events $m$ in an interval of duration $T, m=N_{T}(t)$, are

$$
\langle m\rangle=\mu T
$$

and

$$
\operatorname{Var}(m)=\langle m\rangle+\rho\langle m\rangle^{2} / M_{e},
$$

respectively. This expression is cast in a familiar form with $M_{e}$ behaving like a degrees-of-freedom parameter, given by

$$
M_{e}^{-1}=\frac{2}{T} \int_{0}^{T}\left(1-\frac{\tau}{T}\right) \gamma(\tau) \mathrm{d} \tau .
$$

The quantity

$$
\tau_{\rho}=2 \int_{0}^{\infty} \gamma(\tau) \mathrm{d} \tau
$$

represents the width of the function $\gamma(\tau)$. It can be shown that

$$
\tau_{e}=\frac{\langle\tilde{\tau}\rangle}{\rho}\left[\frac{\operatorname{Var}(\tilde{\tau})}{\langle\tilde{\tau}\rangle^{2}}-1\right] .
$$

From Eqs. (A8) and (A9), it follows that

$$
\begin{array}{ll}
M_{e} \approx 1, & T \ll \tau_{e}, \\
M_{e} \approx T / \tau_{e}, & T \gg \tau_{e} .
\end{array}
$$

\section{Filtered Renewal Processes}

Let

$$
X(t)=\sum_{k} h\left(t-t_{k}\right)
$$

be a filtered renewal process, where the $\left\{t_{k}\right\}$ are realizations of a renewal point process and $h(t)$ is an arbitrary impulseresponse function. The mean and the autocorrelation function of $X(t)$ are then easily shown to be

$$
\langle X(t)\rangle=\mu \int_{-\infty}^{\infty} h(t) \mathrm{d} t
$$

and

$$
\begin{aligned}
& \left\langle X^{*}(t) X(t+\tau)\right\rangle \\
& \quad=\left\langle X^{*}(t)\right\rangle\langle X(t+\tau)\rangle+\mu \bar{h}(\tau)+\mu^{2} \rho \gamma(\tau) \circledast \bar{h}(\tau),
\end{aligned}
$$

respectively. The symbol $\circledast$ represents the convolution, and

$$
\bar{h}(\tau) \equiv \int_{-\infty}^{\infty} h^{*}(t) h(t+\tau) \mathrm{d} t .
$$

\section{Filtered Marked Renewal Processes}

Let $X_{j}(t)=\Sigma_{k} h_{j}\left(a_{k}, t-t_{k}\right)$, with $j=1,2$, be two filtered renewal processes, where the $\left\{t_{k}\right\}$ are described by a renewal point process and where $\left\{a_{k}\right\}$ are independent and identically distributed random variables. Then

$$
\left\langle X_{j}(t)\right\rangle=\mu \int_{-\infty}^{\infty}\left\langle h_{j}(a, t)\right\rangle_{a} \mathrm{~d} t, \quad j=1,2
$$

and

$$
\begin{aligned}
& \left\langle X_{1}^{*}(t) X_{2}(t+\tau)\right\rangle \\
& \quad=\left\langle X_{1} *(t)\right\rangle\left\langle X_{2}(t)\right\rangle+\mu \int_{-\infty}^{\infty}\left\langle h_{1} *(a, t) h_{2}(a, t+\tau)\right\rangle_{a} \mathrm{~d} t \\
& +\mu^{2} \rho \gamma(\tau) \circledast \int_{-\infty}^{\infty}\left\langle h_{1} *(a, t)\right\rangle_{a}\left\langle h_{2}(a, t+\tau)\right\rangle_{a} \mathrm{~d} t, \quad(\mathrm{~A} 17)
\end{aligned}
$$

in analogy with Eqs. (A13) and (A14) above.

\section{E. Examples}

1. Exponentially Distributed Interevent Times

This is the simplest of all renewal processes. The intereventtime probability density is

$$
f(\tilde{\tau})=\mu e^{-\mu \tilde{\tau}} U(\tilde{\tau}), \quad U(\tilde{\tau})=\left\{\begin{array}{ll}
1, & \tilde{\tau} \geqslant 0 \\
0, & \tilde{\tau}<0
\end{array} .\right.
$$

Furthermore,

$$
\rho=0
$$

and

$$
\operatorname{Var}(m)=\langle m\rangle .
$$


The results are, of course, familiar since the process is Poisson. A correlation time cannot be defined for this process because it has zero memory (the covariance is a $\delta$ function); the characteristic time between events is simply the inverse of its rate, i.e., $1 / \mu$.

\section{Gamma-Distributed Interevent Times}

In this case

$$
f(\tilde{\tau})=\frac{(\mu \mathcal{N})^{\mathcal{N}} \tilde{\tau}^{\mathcal{N}-1}}{(\mathcal{N}-1) !} e^{-\mu \mathcal{N} \tilde{\tau}} U(\tilde{\tau}),
$$

where $\mathcal{N}$ is a parameter representing the order of the distribution. We shall refer to this as the gamma- $\mathcal{N}$ renewal process. As is well known, it may be generated from a Poisson point process by the selection of every $\mathcal{N}$ th event. ${ }^{19}$ There does not appear to be a formula available for $\gamma(\tau)$ in the case of arbitrary $\mathcal{N}$. However, we have proved the following results:

$$
\begin{aligned}
\rho & =-1, \\
\gamma(\tau) & =\exp (-4 \mu|\tau|), \quad \mathcal{N}=2, \\
\gamma(\tau) & =[\exp (-8 \mu|\tau|)+\exp (-4 \mu|\tau|) \sin (4 \mu|\tau|)], \quad \mathcal{N}=4, \\
\tau_{e} & =\left(1-\frac{1}{\mathcal{N}}\right) / \mu .
\end{aligned}
$$

The Laplace transform of $\gamma(\tau)$, on the other hand, is available for arbitrary $\mathcal{N}$. It is given by

$$
\begin{aligned}
& \bar{\gamma}(s)=\frac{1}{s}-\frac{1}{\mu} \bar{Q}(s), \\
& \bar{Q}(s)=\left[(1+s / \mathcal{N} \mu)^{\mathcal{N}}-1\right]^{-1} .
\end{aligned}
$$

For $T \gg \tau_{e}$, the asymptotic count variance and the Fano factor become ${ }^{19}$

$$
\begin{aligned}
\operatorname{Var}(m) & \approx\langle m\rangle / \mathcal{N}, \\
F_{m} & \approx 1 / \mathcal{N},
\end{aligned}
$$

whereas for $T \ll \tau_{e}$

$$
\begin{aligned}
\operatorname{Var}(m) & \approx\langle m\rangle, \\
F_{m} & \approx 1 .
\end{aligned}
$$

Thus, for $\mathcal{N}>1$, the counting distribution for the process is sub-Poisson. If the process is turned on at $t=0$, the probability distribution of $m$ is given by

$$
p(m)=\sum_{k=\mathcal{N} m}^{\mathcal{N} m+\mathcal{N}-1} \frac{(\mathcal{N} \mu T)^{k}}{k !} e^{-\mathcal{N} \mu T} .
$$

The gamma-distributed interevent-time process may be generalized by allowing $\mathcal{N}$ to take on continuous, rather than integer, values. This is accomplished by using the continuous extension $(n-1) ! \rightarrow \Gamma(n)$. In this case $0<\mathcal{N}<1$ corresponds to a super-Poisson gamma- $\mathcal{N}$ renewal process.

\section{Dead-Time-Modified Poisson Process}

For a nonparalyzable dead-time-modified Poisson point process $^{12,19}$

$$
f(\tilde{\tau})=\lambda \exp \left[-\lambda\left(\tilde{\tau}-\tau_{d}\right)\right] U\left(\tilde{\tau}-\tau_{d}\right),
$$

with

$$
\lambda=\mu /\left(1-\mu \tau_{d}\right) .
$$

The quantity $\tau_{d}$ is a parameter (dead time), and $D=\lambda \tau_{d}$. It can be shown that

$$
\begin{aligned}
\rho= & -1, \\
\gamma(\tau)= & 1-\frac{1+\lambda \tau_{d}}{\lambda} \sum_{l=1}^{\infty} \frac{\left[\lambda\left(\tau-l \tau_{d}\right)\right]^{l-1}}{(l-1) !} \\
& \times \exp \left[-\lambda\left(\tau-l \tau_{d}\right)\right] U\left(\tau-l \tau_{d}\right), \quad \tau \geq 0
\end{aligned}
$$

and

$$
\tau_{e}=\tau_{d}\left(2-\mu \tau_{d}\right)
$$

For $T \gg \tau_{e}$,

$$
\begin{aligned}
\operatorname{Var}(m) & \approx\langle m\rangle /\left(1+\lambda \tau_{d}\right)^{2}=\langle m\rangle\left(1-\mu \tau_{d}\right)^{2}, \\
F_{m} & \approx\left(1-\mu \tau_{d}\right)^{2},
\end{aligned}
$$

whereas for $T \ll \tau_{e}$,

$$
\begin{aligned}
\operatorname{Var}(m) & \approx\langle m\rangle . \\
F_{m} & \approx 1 .
\end{aligned}
$$

This process is always sub-Poisson. Of course, in the limit as $\tau_{d} \rightarrow 0$, it approaches the Poisson point process. The counting distribution has been discussed in detail elsewhere. ${ }^{12}$

\section{Pulse Train of Random Phase}

Finally, we consider the deterministic interevent time density

$$
f(\tilde{\tau})=\delta\left(\tilde{\tau}-T_{0}\right),
$$

which corresponds to a periodic pulse train. We assume that the pulse train has random phase to ensure the stationarity of the process. This process has the following properties:

$$
\begin{aligned}
\rho & =-1, \\
\gamma(\tau) & =1-T_{0} \sum_{l \neq 0} \delta\left(\tau-l T_{0}\right) .
\end{aligned}
$$

Furthermore,

$$
\operatorname{Var}(m)=\theta_{0}\left(1-\theta_{0}\right),
$$

with

$$
\theta_{0}=T / T_{0}-L
$$

where $L$ is the largest integer that is smaller than $T / T_{0}$. As $T / T_{0}$ increases, it is clear that $\langle m\rangle$ increases, while $\operatorname{Var}(m)$ is constrained to be $<0.25$. Thus the index of dispersion, $\operatorname{Var}(m) /\langle m\rangle$, decreases toward 0 as $\langle m\rangle$ increases. The counting distribution approaches a delta function in this limit. The periodic pulse train with random phase is the limit of many renewal processes, such as the gamma- $\mathcal{N}$ as $\mathcal{N} \rightarrow \infty$ and the nonparalyzable dead-time-modified Poisson process as $\lambda \tau_{d} \rightarrow \infty$.

\section{ACKNOWLEDGMENT}

This research was supported by the Joint Services Electronics Program at Columbia University and by the National Science Foundation. 
B. E. A. Saleh is also with the Department of Electrical and Computer Engineering, University of Wisconsin, Madison, Wisconsin 53706.

J. Peřina was I. I. Rabi Visiting Scientist at Columbia University, supported by the Alfred P. Sloan Foundation. His permanent address is Laboratory of Optics, Palacký University, Olomouc, Czechoslovakia.

\section{REFERENCES}

1. D. R. Cox, "Some statistical methods connected with series of events," R. Stat. Soc. J. B 17, 129-164 (1955).

2. R. Hanbury-Brown and R. Q. Twiss, "Correlation between photons in two coherent beams of light," Nature 177, 27-29 (1956); E. M. Purcell, "Correlation in the fluctuations of two photoelectric currents evoked by coherent beams of light," Nature 178, 1449-1450 (1956); L. Mandel, "Fluctuations of photon beams: The distribution of the photo-electrons," Proc. Phys. Soc. London 74, 233-243 (1959); M. C. Teich and B. E. A. Saleh, "Fluctuation properties of multiplied-Poisson light: measurement of the photon-counting distribution for radioluminescence radiation from glass," Phys. Rev. A 24, 1651-1654 (1981).

3. R. J. Glauber, "The quantum theory of optical coherence," Phys. Rev. 130, 2529-2539 (1963); "Coherent and incoherent states of the radiation field," Phys. Rev. 131, 2766-2788 (1963).

4. D. Stoler, "Photon antibunching and possible ways to observe it," Phys. Rev. Lett. 33, 1397-1400 (1974).

5. M. C. Teich, B. E. A. Saleh, and D. Stoler, "Antibunching in the Franck-Hertz Experiment," Opt. Commun. 46, 244-248 (1983).

6. D. Stoler, "Equivalence classes of minimum uncertainty packets," Phys. Rev. D 1, 3217-3219 (1970).

7. U. M. Titulaer and R. J. Glauber, "Correlation functions for coherent fields," Phys. Rev. 140, B676-B682 (1965); "Density operators for coherent fields," Phys. Rev. 145, 1041-1050 (1966).

8. M. C. Teich, P. R. Prucnal, G. Vannucci, M. E. Breton, and W. J. McGill, "Multiplication noise in the human visual system at threshold: 3. The role of non-Poisson fluctuations," Biol. Cybern. 44, 157-165 (1982).

9. Recent work has been summarized in V. Peřinová and J. Peřina, "Quantum statistics of quadratic optical parametric processes with intense coherent stochastic pumping," Opt. Acta 28, 769-793 (1981).

10. N. Tornau and A. Bach, "Quantum statistics of two-photon absorption," Opt. Commun. 11, 46-49 (1974); H. D. Simaan and R. Loudon, "Quantum statistics of single-beam two-photon absorption," J. Phys. A 8, 539-554 (1975); H. Paul, U. Mohr, and W. Brunner, "Change of photon statistics due to multi-photon absorption," Opt. Commun. 17, 145-148 (1976); A. Bandilla and H. H. Ritze, "Einfluss einer inneren zwei-photonen-Absorptionszelle auf die Photonenstatistik eines ein-moden-Lasers," Opt. Commun. 19, 169-171 (1976); H. D. Simaan, "A master equation approach to the hyper-Raman effect," J. Phys. A. 11, 1799-1802 (1978); P. D. Drummond, K. J. McNeil, and D. F. Walls, "Bistability and photon antibunching in sub/second harmonic generation," Opt. Commun. 28, 255-258 (1979); C. W. Helstrom, "Maximum photon antibunching in generalized coherent states," Opt. Commun. 28, 363-364 (1979); C. Sibilia and M. Bertolotti, "The photon anticorrelation effect in the stimulated annihilation process of electron-positron pairs," Opt. Acta 28, 503-513 (1981); W. Becker, M. O. Scully, and M. S. Zubairy, "Generation of squeezed coherent states via a free-electron laser," Phys. Rev. Lett. 48, 475-477 (1982); L. A. Lugiato, F. Casagrande, and L. Pizzuto, "Fluctuation theory in quantum-optical systems," Phys. Rev. A 26, 3438-3458 (1982); D. F. Walls, "Quantum fluctuations of the electromagnetic field," Appl. Phys. B 28, 108-109 (1982); J. Peřina, V. Peřinová, and J. Kodousek, "On the relations of antibunching, sub-Poissonian statistics and squeezing," Opt. Commun. 49, 210-214 (1984). See also R. Loudon, "Non-classical effects in the statistical properties of light," Rep. Prog. Phys. 43, 913-949 (1980), and references therein, for a discussion of nonclassical single- and double-beam experiments. Recent reviews have been presented by M. Schubert and B. Wilhelmi, "The mutual dependence between coherence properties of light and nonlinear optical processes," in Progress in Optics, E. Wolf, ed. (North-Holland, Amsterdam, 1980), Vol. 17, pp. 163-238; by J. Peřina, "Photocount statistics of radiation propagating through random and nonlinear media," in Progress in Optics, E. Wolf, ed. (North-Holland, Amsterdam, 1980), Vol. 18, pp. 127-203; and by H. Paul, "Photon antibunching," Rev. Mod. Phys. 54, 1061-1102 (1982).

11. H. J. Kimble, M. Dagenais, and L. Mandel, "Photon antibunching in resonance fluorescence," Phys. Rev. Lett. 39, 691-695 (1977); H. J. Kimble, M. Dagenais, and L. Mandel, "Multiatom and transit-time effects on photon-correlation measurements in resonance fluorescence," Phys. Rev. A 18, 201-207 (1978); M. Dagenais and L. Mandel, "Investigation of two-time correlations in photon emissions from a single atom," Phys. Rev. A 18, 2217-2228 (1978); J. D. Cresser, J. Häger, G. Leuchs, M. Rateike, and $\mathrm{H}$. Walther, "Resonance fluorescence of atoms in strong monochromatic laser fields," in Dissipative Systems in Quantum Optics, Vol. 27 of Topics in Current Physics, R. Bonifacio, ed. (Springer-Verlag, Berlin, 1982), pp. 21-59.

12. P. R. Prucnal and M. C. Teich, "Refractory effects in neural counting processes with exponentially decaying rates," IEEE Trans. Syst. Man Cybern. SMC-13, 1028-1033 (1983); B. I. Cantor and M. C. Teich, "Dead-time-corrected photocounting distributions for laser radiation,” J. Opt. Soc. Am. 65, 786-791 (1975).

13. L. Mandel, "Sub-Poissonian photon statistics in resonance fluorescence," Opt. Lett. 4, 205-207 (1979).

14. R. J. Cook, "Photon number statistics in resonance fluorescence," Phys. Rev. A 23, 1243-1250 (1981).

15. R. Short and L. Mandel, "Observation of sub-Poissonian photon statistics,” Phys. Rev. Lett. 51, 384-387 (1983).

16. H. J. Carmichael and D. F. Walls, "Proposal for the measurement of the resonant Stark effect by photon correlation techniques," J. Phys. B 9, L43-L46 (1976); H. J. Carmichael and D. F. Walls, "Quantum-mechanical master equation treatment of the dynamic Stark effect," J. Phys. B. 9, 1199-1219 (1976); H. J. Carmichael, P. Drummond, P. Meystre, and D. F. Walls, "Intensity correlations in resonance fluorescence with atomic number fluctuations," J. Phys. A 11, L121-L126 (1978); E. Jakeman, E. R. Pike, P. N. Pusey, and J. M. Vaughan, "The effect of atomic number fluctuations on photon antibunching in resonance fluorescence," J. Phys. A 10, L257-L259 (1977).

17. B. E. A. Saleh, D. Stoler, and M. C. Teich, "Coherence and photon statistics for optical fields generated by Poisson random emissions," Phys. Rev. A 27, 360-374 (1983).

18. M. C. Teich, "Role of the doubly stochastic Neyman Type-A and Thomas counting distributions in photon detection," Appl. Opt. 20, 2457-2467 (1981); B. E. A. Saleh and M. C. Teich, "Multiplied-Poisson noise in pulse, particle, and photon detection," Proc. IEEE 70, 229-245 (1982).

19. D. R. Cox, Renewal Theory (Methuen, London, 1962); E. Parzen, Stochastic Processes (Holden-Day, San Francisco, Calif., 1962).

20. M. C. Teich and B. E. A. Saleh, "Antibunched and sub-Poisson light from the Franck-Hertz experiment," J. Opt. Soc. Am. 73, 1876 (A) (1983).

21. M. C. Teich and B. E. A. Saleh, "Effects of random deletion and additive noise on bunched and antibunched photon-counting statistics," Opt. Lett. 7, 365-367 (1982). In this reference, the definition of antibunching was taken to be identical with that of sub-Poisson. The definition of antibunching used in the current work $\left[g^{(2)}(0)<1\right]$ is in accord with the more usual usage of the term.

22. J. Peřina, B. E. A. Saleh, and M. C. Teich, "Independent photon deletions from quantized boson fields: the quantum analog of the Burgess variance theorem," Opt. Commun. 48, 212-214 (1983).

23. A. T. Forrester, "Optical-field effects produced by fluctuations of the density of excited states," J. Opt. Soc. Am. 62, 654-658 (1972).

24. R. Loudon, "Non-classical effects in the statistical properties of light," Rep. Prog. Phys. 43, 913-949 (1980).

25. D. W. Schaefer and P. N. Pusey, "Statistics of non-Gaussian scattered light," Phys. Rev. Lett. 29, 843-845 (1972). 
26. M. Born and E. Wolf, Principles of Optics, 6th ed. (Pergamon, Oxford, 1980).

27. L. Mandel, "Photon interference and correlation effects produced by independent quantum sources," Phys. Rev. A 28, 929-943 (1983).

28. B. E. A. Saleh, Photoelectron Statistics (Springer-Verlag, Berlin, 1978).

29. J. Peřina, Coherence of Light, 2nd ed. (Reidel, Dordrecht, The Netherlands, 1984).

30. D. R. Cox and V. Isham, Point Processes (Chapman and Hall, London, 1980).

31. M. M. Miller and E. A. Mishkin, "Anti-correlation effects in quantum optics," Phys. Lett. 24A, 188-189 (1967).

32. N. B. Abraham and S. R. Smith, "Stimulated versus spontaneous emission as a cause of photon correlations," Phys. Rev. A 15, 421-428 (1977).

33. S. Friberg and L. Mandel, "Coherence properties of the linear photon amplifier," Opt. Commun. 46, 141-148 (1983).

34. A. Y. Khinchine, Mathematical Methods in the Theory of Queuing (Griffin, London, 1960).

35. D. R. Cox and W. L. Smith, "The superposition of several strictly periodic sequences of events," Biometrika 40,1-11 (1953).

36. D. R. Cox and W. L. Smith, "On the superposition of renewal processes," Biometrika 41, 91-99 (1954).

37. E. Çinlar, "Superposition of point processes," in Stochastic Point Processes, P. A. W. Lewis, ed. (Wiley-Interscience, New York, 1972), pp. 549-606. 